\title{
ETGraph: A Graph-Based Approach for Visual Analytics of Eye-Tracking Data
}

\author{
Yi Gu ${ }^{\mathrm{a}}$, Chaoli Wang ${ }^{\mathrm{b}}$, Robert Bixler ${ }^{\mathrm{a}}$, Sidney D’Mello ${ }^{\mathrm{a}, \mathrm{b}}$ \\ ${ }^{a}$ Department of Computer Science and Engineering, University of Notre Dame, Notre Dame, IN 46556 \\ ${ }^{b}$ Department of Psychology, University of Notre Dame, Notre Dame, IN 46556
}

\begin{abstract}
Mind wander(ing) (MW) or zoning out is a ubiquitous phenomenon where attention involuntary shifts from task-related processing to task-unrelated thoughts. Unfortunately, MW is a highly internal state so it cannot be readily inferred from overt behaviors and expressions. To help experts investigate mind wanderings, we present a graph-based approach for visual analytics of eye-tracking data, which utilizes the graph representations to illustrate the reading patterns and further help experts detect and verify mind wanderings based on the graph structures and other graph attributes. The input data are collected from multiple participants reading multiple pages of a book on a computer screen. Our approach first clusters fixations into fixation clusters, then creates the eyetracking graph, i.e., ETGraph, for use in conjunction with the standard page view, time view, and statistics view. The graph view presents a visual representation of the actual reading patterns of a single participant or multiple participants and therefore serves as the main visual interface for exploration and navigation. We design a suite of techniques to help users identify common reading patterns and outliers for analytical reasoning at three different levels of detail: single participant single page, single participant multiple pages, and multiple participants single page. Interactive querying and filtering functions are provided for reducing visual clutter in the visualization and enabling users to answer questions and glean insights. Our tool also facilitates the detection and verification of mind wandering that the experts seek to investigate. We conduct a user study and an expert evaluation to assess the effectiveness of ETGraph in terms of its visual summarization and comparison capabilities.
\end{abstract}

Keywords: Eye-tracking data, Visual analytics, Graph layout, Saccade outlier detection, Repeated scanpath detection, Participant comparison and clustering

\section{Introduction}

2 With advances of the eye-tracking technology, eye-trackers 3 are getting increasingly affordable for use in research and ed4 ucation. In this work, we study eye-tracking data collected 5 from multiple participants reading multiple pages of a book on 6 a computer screen. A research group led by a cognitive sci7 entist collected the data in order to investigate cognitive pro8 cesses during reading. In this paper, we focus on attentional ${ }_{9}$ lapses called mind wandering, but our solution can be applied 10 to investigate other cognitive and affective phenomena, such as ${ }_{11}$ cognitive load, inference generation, boredom, and so on.

12 Mind wander(ing) (MW) or zoning out is a ubiquitous phe13 nomenon where attention involuntary shifts from task-related ${ }_{14}$ processing to task-unrelated thoughts [1]. Considerable research ${ }_{15}$ over the last 5-10 years has documented the widespread inci${ }_{16}$ dence and negative consequence of MW both in the lab and 17 in the real world. In one highly-cited, large-scale study, MW 18 was tracked in 5,000 individuals from 83 countries working in 1986 occupations with an iPhone app that prompted people to re${ }_{20}$ port MW at random intervals throughout the day [2]. People ${ }_{21}$ reported MW for $46.9 \%$ of the prompts, which confirmed nu${ }_{22}$ merous lab studies on the pervasiveness of MW [3, 4]. MW ${ }_{23}$ is also more than merely incidental as a recent meta-analysis ${ }_{24} \mathrm{Of} 88$ studies indicated a negative correlation between MW and ${ }_{25}$ performance across a variety of tasks [5], a correlation which ${ }_{26}$ increases in proportion to task complexity. MW occurs around
${ }_{27} 30 \%$ of the time during reading and is negatively correlated with 28 reading comprehension.

Unfortunately, MW is a highly internal state so it cannot so be readily inferred from overt behaviors and expressions [6]. ${ }_{31}$ Thus, the most common way to measure MW is via self-report. ${ }_{32}$ Self-caught methods ask people to monitor their attentional lev${ }_{33}$ els and to indicate (e.g., by pressing a key) when they catch 34 themselves MW. For example, a participant in a reading study 35 may be asked to press a key when they realize that "they have ${ }_{36}$ no idea what they just read because they were thinking about ${ }_{37}$ something else altogether" [7]. The same instructions are used ${ }_{38}$ in probe-caught methods; however, participants are prompted ${ }_{39}$ (e.g., via an auditory probe) at multiple intervals to indicate if ${ }_{40}$ they are MW at the time of the probe [8]. MW data collected ${ }_{41}$ in this fashion have shown predictable relationships with phys${ }_{42}$ iology [9], pupillometry [10], eye gaze [7], and task perfor43 mance [5], thereby providing some validity for this measure44 ment approach. However, there are many limitations of self${ }_{45}$ report measures, so it would be beneficial to obtain behavioral ${ }_{46}$ indicators of MW. In this paper, we focus on eye gaze to track ${ }_{47} \mathrm{MW}$, which is motivated by decades of scientific evidence in ${ }_{48}$ support of an eye-mind link that suggests a tight coupling be${ }_{49}$ tween internal thoughts and eye movements [11]. Our goal in ${ }_{50}$ this work is to design a visual interface that helps researchers ${ }_{51}$ investigate reading patterns (adduced from eye-movements) as${ }_{52}$ sociated with MW. Our long-term goal is to use these expert 
${ }_{53}$ insights to improve automated measures of MW, which are still 54 in their infancy $[12,13]$.

${ }_{55}$ We restrict our attention to the reading study with static ${ }_{56}$ stimuli (i.e., static text on screen) and aim to investigate reading ${ }_{57}$ behaviors for tens of participants. In a recent article, Raschke ${ }_{58}$ et al. [14] pointed out that visually analyzing multiple viewers 59 with an individual stimulus is an interesting research topic. It 60 is also challenging to present an effective solution to find pat${ }_{61}$ terns, detect outliers, and compare different participants. The ${ }_{62}$ key issue is how to design a visual analytics tool that leverages ${ }_{63}$ different visual mappings, interfaces and interactions to facili64 tate visual exploration, navigation and comparison of the vast 65 amount of eye-tracking data.

66 Our main contribution lies in the designing of a visual ana${ }_{67}$ lytics framework that helps researchers investigate reading pat68 terns, which could be further categorized into three different 69 levels of detail: SPSP (single participant single page), SPMP 70 (single participant multiple pages), and MPSP (multiple partic71 ipants single page). For SPSP, our visual interface allows re72 searchers to capture the normal and abnormal reading patterns ${ }_{73}$ of a participant on a single page and identify possible MWs. ${ }_{74}$ This may be used to improve the automated measures of MWs. 75 For SPMP, our visual interface helps researchers identify simi${ }_{76}$ lar behaviors among continuous pages. For MPSP, the common 77 reading patterns of the same page from all participants are illus78 trated. In addition, we allow users to compare the differences 79 between any two selected participants.

To this end, we propose to transform the eye-tracking data ${ }_{81}$ gathered from a reading study into a graph view for visual ana82 lytics. Graph-based representations have been utilized for eye83 tracking data analysis. For instance, Tory et al. [15] studied 84 the relation between areas of interest (AOIs) using a directed ${ }_{85}$ graph visualization. In such a graph, each node represents one ${ }_{86} \mathrm{AOI}$ and an edge connecting two nodes represents their tran87 sition. The edge thickness depicts the number of transitions ${ }_{88}$ between the two AOIs. In their work, the graph view was used 89 mainly for a visual overview but not for interactive exploration. ${ }_{90}$ In contrast, our work is pitched at a finer level of detail. That ${ }_{91}$ is, instead of using AOIs for visual summarization, we group ${ }_{92}$ fixations into clusters and build a graph, i.e., ETGraph (eye${ }_{93}$ tracking graph), to support interactive examination of the un${ }_{94}$ derlying structure in the eye-tracking data. Multiple coordi95 nated views are utilized to dynamically link the graph view with ${ }_{96}$ the standard page view during the interaction.

We design a suite of techniques to help users identify com98 mon reading patterns and outliers for analytical reasoning at ${ }_{99}$ different levels of detail. Our tool enables visual comparison 100 of different pages being read by a single participant as well as 101 when the same page is read by different participants. It also 102 supports a global overview of reading patterns of all pages by ${ }_{103}$ all participants and local exploration of a single page being read 104 by a single participant. We demonstrate the effectiveness of our 105 approach by showing experimental results gathered from ana106 lyzing the eye-tracking data. We also report the feedback of 107 using our tool for visual exploration and MW investigation.

\section{2. Related Work}

Rayner [11] synthesized over 100 years of eye-tracking re110 search and conducted an excellent survey of eye-tracking ap111 plications in reading and other information processing tasks. ${ }_{112}$ Duchowski [16] presented a breadth-first survey of eye-tracking 113 applications in the following domains: neuroscience, psychol114 ogy, industrial engineering and human factors, marketing or ad115 vertising, and computer science. Recently, Blascheck et al. [17] ${ }_{116}$ presented a comprehensive state-of-the-art report on techniques 117 for visualizing eye-tracking data. They classified the visualiza118 tion techniques into different categories based on properties of 119 eye-tracking data and properties of visualization techniques.

Tracking eye-movement leads to vast amounts of fixation 121 points and scanpaths which can be clustered and visualized for 122 clear observation of patterns or outliers. Santella and DeCarlo [18] ${ }_{123}$ presented a robust clustering of eye-movement recordings using 124 the mean-shift method, which forms a structured representation 125 of the viewer's attention and avoids heavy influence from noise 126 or outliers. S̆pakov and Räihä [19] introduced EiKV, which ${ }_{127}$ shows the reading and typing processes in parallel with details 128 for each word presented in word bars so that users could iden129 tify the unusual events. Goldberg and Helfman [20] proposed a 130 solution to identify scanning strategies by automatically aggre${ }_{131}$ gating groups of matching scanpaths. First, they converted each ${ }_{132}$ scanpath into a sequence of AOIs visited in order. Sequences of ${ }_{133}$ AOIs were concatenated into one sequence and plotted with a ${ }_{134}$ dotplot. Then they used linear repeated scanpaths to find match135 ing sequences in the dotplot for clustering the scanpaths hierar${ }_{136}$ chically. Tang et al. [21] designed EyeMap, a system which 137 supports word segmentation, eye movement data visualization, 138 and XML data format. Since word segmentation could identify 139 separated words so that fixations are mapped to the words, Eye${ }_{140}$ Map could support writing systems using different languages. ${ }_{141}$ Furthermore, gaze, scanpath, and statistics information are dis142 played to support various kinds of queries. In addition, the ${ }_{143} \mathrm{XML}$ data format is utilized for describing data from a wide ${ }_{144}$ range of reading experiments for data export and sharing.

145 To visualize the spatiotemporal behaviors of eye-movement ${ }_{146}$ data, one can use heat maps or gaze plots. However, these vi${ }_{147}$ sual representations suffer from high aggregation (heat maps) 148 and overplotting (gaze plots). New visual mappings and repre149 sentations are needed for investigating the vast amounts of spa150 tiotemporal eye gaze trajectories. Tsang et al. [22] presented 151 eSeeTrack, an eye-tracking visualization prototype to facilitate 152 the exploration and comparison of sequential gaze orderings in 153 a static or dynamic scene. Their work integrates a timeline and 154 a tree-structured representation to encode multiple aspects (du155 ration, frequency, and fixation ordering) of eye-tracking data. 156 Burch et al. [23] transformed eye-movement data into a dy157 namic graph and achieved a fair tradeoff between aggregation 158 and details. Their dynamic graph is a sequence of static graphs 159 where nodes represent AOIs and directed edges show transi160 tions between source and target AOIs. Burch et al. [24] de${ }_{161}$ signed AOI Rivers for investigating time-varying fixation fre${ }_{162}$ quencies, transitions between AOIs, and the sequential order ${ }_{163}$ of gaze visits to AOIs. Based on the ThemeRiver technique, 
164 they represented the trajectory data as time-varying river-like 165 structures enhanced by influents, effluents, and AOIs transi166 tions, similar to Sankey diagrams.

167 Beyond analyzing eye-tracking data, eye-movement analy168 sis has gained its popularity as a tool for evaluating visualiza169 tion research. Andrienko et al. [25] proposed a visual analytics 170 methodology originated from analysis of geographic data for 171 analyzing large amounts of eye-tracking data. They focused on 172 deriving common task solution strategies for a given static stim- ${ }^{217}$ 173 ulus shown to participants. Their work presents a systematic 218 174 evaluation of movement analysis methods for the applicabil- ${ }^{219}$ 175 ity of eye-tracking data and provides the guidelines for choos- 220 176 ing appropriate methods given the analysis goals. Blascheck et 221 177 al. [26] presented a visual analytics approach for an integrated ${ }_{222}$ 178 analysis of multiple concurrent evaluation procedures such as ${ }_{223}$ 179 measures of task performance, think-aloud protocols, analysis 180 of interaction logs, and eye tracking. An efficient exploratory 224 ${ }_{181}$ search and reasoning process is supported through automatic ${ }_{182}$ pattern finding to derive common eye-interaction-thinking pat- 225 183 terns between participants.

\section{3. Research Questions}

185 Blascheck et al. [17] defined the basic terminology related 186 to eye-tracking data. We briefly introduce several of them that 187 are used in this work. First of all, gaze points are the raw eye188 tracking data and each fixation is an aggregation of gaze points 189 based on specified area and timespan. Furthermore, saccades 190 describe a rapid eye movement from one fixation to another, 191 and a scanpath is a sequence of alternating fixations and sac192 cades. Analyzing them would help users understand the eye193 movements, therefore, there are a lot of related research ques194 tions. Since our eye-tracking data are gathered from multi195 ple participants reading multiple pages of a book, we propose 196 the following research questions categorized into three different 197 levels of detail: SPSP (single participant single page), SPMP 198 (single participant multiple pages), and MPSP (multiple partic199 ipants single page). The questions associated with SPSP, SPMP, 200 and MPSP focus on the reading patterns of a participant read201 ing one page, the consistency of a participant reading multiple 202 pages, and the behavior similarities/differences between partic203 ipants, respectively.

- SPSP (single participant single page):

- Q1. What is the scanpath structure of each participant when reading a single page?

- Q2. Does the participant exert a different amount of effort reading different parts of the page?

- Q3. Does the scanpath involve forward and/or backward saccade outliers (i.e., saccades with amplitudes larger than a given threshold)? If yes, when and where do these saccade outliers occur and how frequent are they? Does the same saccade outlier occur multiple times?

- Q4. Does the scanpath involve repeated scanpaths (i.e., a scanpath that represents rereading previously

\begin{tabular}{|c|c|c|c|c|c|c|}
\hline & C1 & C2 & C3 & C4 & C5 & C6 \\
\hline SPSP & Q1 & Q3 & Q4 & Q5 & - & Q2 \\
\hline SPMP & Q6 & - & - & - & - & Q7 \\
\hline MPSP & Q8 & - & - & - & Q10 & Q9 \\
\hline
\end{tabular}

Table 1: The ten research questions Q1 $\sim$ Q10 associated with SPSP (single participant single page), SPMP (single participant multiple pages), and MPSP (multiple participants single page) are classified into six categories $\mathbf{C 1} \sim \mathbf{C 6}$.

read text along the same path)? If yes, when and where do these repeated scanpaths occur and how frequent are they? Does the appearance of saccade outliers have any correlation with the appearance of repeated scanpaths?

- Q5. Does the participant MW on a page? If yes, when and where does MW occur?

- SPMP (single participant multiple pages):

- Q6. Is the reading pattern of a participant consistent across all pages?

- Q7. What are the temporal dynamics of reading behavior across consecutive pages? For example, do the saccade outliers on the current page have a relationship with the saccade outliers on the next page?

- MPSP (multiple participants single page):

- Q8. What are the common patterns of multiple participants when reading the same page?

- Q9. Do they spend a different amount of time reading different parts of the page?

- Q10. What are the outliers (who, when and where)? Can we cluster participants based on different reading patterns exhibited on the same page?

The research questions can be classified into six categories, as shown in Table 1: C1 scanpath structures $(\mathbf{Q 1}, \mathbf{Q 6}, \mathbf{Q 8}), \mathbf{C 2}$ 242 saccade outliers $(\mathbf{Q 3}), \mathbf{C 3}$ repeated scanpaths $(\mathbf{Q 4}), \mathbf{C 4} \mathrm{MW}$ ${ }_{243}$ (Q5), C5 participant clustering (Q10), and C6 reading efforts ${ }_{244}(\mathbf{Q} 2, \mathbf{Q 7}, \mathbf{Q 9})$. To better answer these questions, we introduce 245 four views as shown in Figure 1: page view, graph view, time 246 view, and statistics view. Categories 1 to 4 can be answered 247 using the graph, page, and time views. Categories 5 and 6 can 248 be answered using the statistics view. In addition, these four 249 views can be combined together to help users better explore 250 and understand the data.

\section{4. ETGraph Construction}

Our goal is to design a visual analytics framework that can 253 help users understand reading patterns of the participants, iden254 tify the anomalous behaviors, and group participants. Specif255 ically, how can we apply the three levels of analysis to obtain 256 new insights on reading patterns? Can we visually discriminate ${ }_{257}$ reading patterns from the graph representations? Can we find 


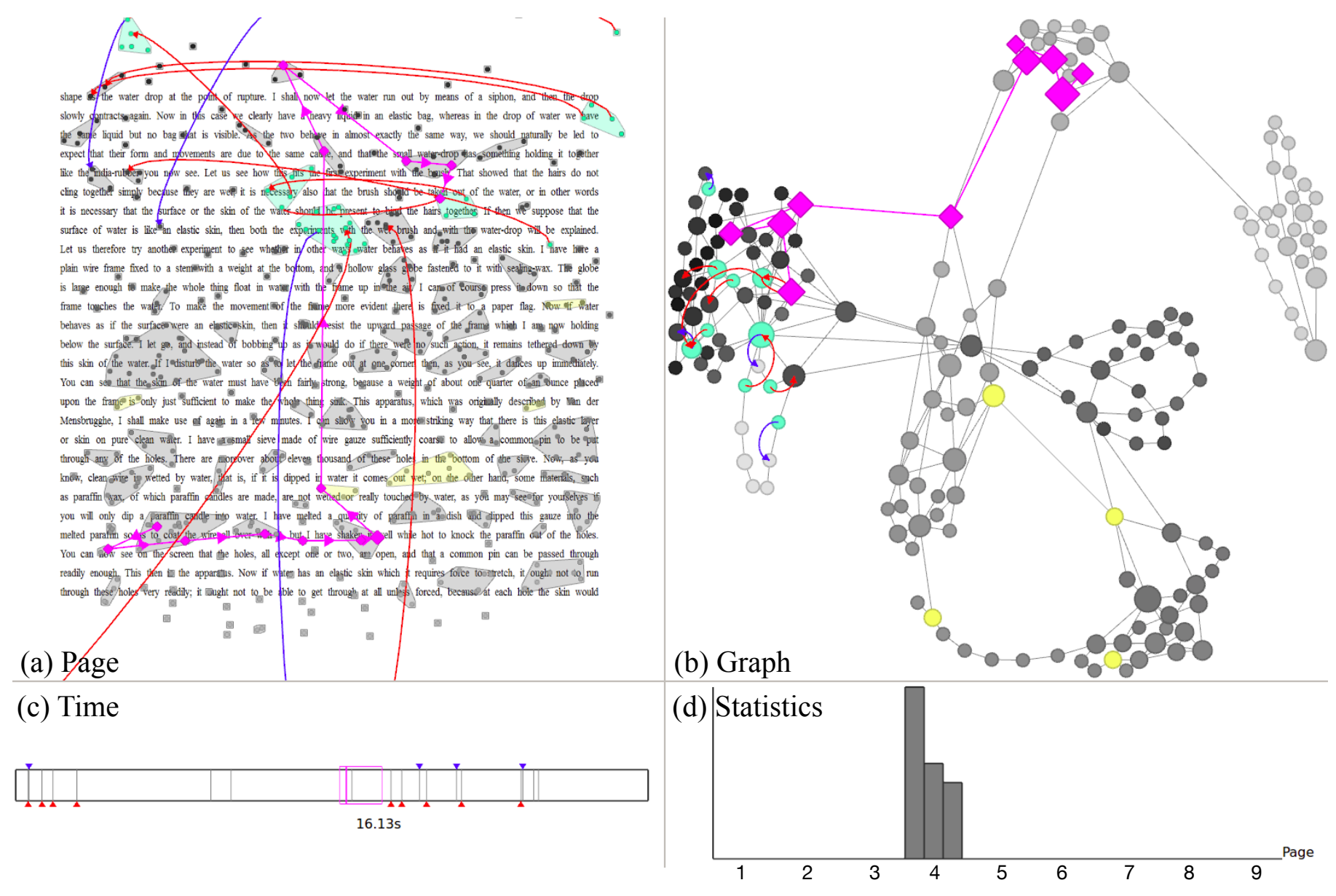

Figure 1: The four views of ETGraph. (a) page view, (b) graph view, (c) time view, and (d) statistics view show saccade outliers and MWs of SPSP. In (a) and (b), fixation clusters and nodes corresponding to saccade outliers are highlighted in yellow. The selected clusters and nodes are in green. Red and blue edges indicate backward and forward saccade outliers, respectively. The fixations and nodes around MW are highlighted as pink diamonds. The arrows between the diamond fixations in the page view shows the scanpaths around MW. In (b), the shading of nodes (from dark to light) indicates the presumed reading order. In (c), the vertical lines indicates the time points that the saccade outliers occurred. Red and blue arrows correspond to the selected saccade outliers. The pink rectangle and the number below show the MW and its duration. (d) shows the distribution of the numbers of saccade outliers in page sections (each page is partitioned into three equal sections: top, middle and bottom).

${ }_{258}$ out the relations between saccade outliers and repeated scan- ${ }_{278}$ 259 paths? Furthermore, can we visually figure out the similari260 ties and/or differences between the reading patterns on different ${ }_{261}$ pages or between different participants? Finally, can we embed 262 the visualization of MWs to help scientists analyze them?

${ }_{263}$ Our strategy to analyze the given eye-tracking data is to 264 first cluster fixations into fixation clusters to produce a coarse${ }_{265}$ level representation of the data. There are two benefits to doing 266 this. First, our fixation clustering provides spatial closeness. ${ }_{267}$ Fixation clusters are spatially close fixations which are similar 268 to gazes as defined in Blascheck et al. [17]. However, unlike ${ }_{269}$ gazes, we do not consider the temporal ordering of fixations 270 when we perform clustering. Second, fixation clusters are not 271 as coarse as AOIs which represent regions of specific interest 272 on the stimulus. Fixation clusters can be created for a single 273 page using fixations from a single participant or by combin274 ing all the fixations from multiple participants. Combining all 275 fixations for clustering would allow us to visualize the reading ${ }_{276}$ patterns of different participants with a common ground of the 277 same fixation clusters.
We propose a graph-based representation for analyzing eye 279 movements using fixation clusters as nodes and a set of sac280 cades as a directed edge between nodes. We call this visual ${ }_{281}$ representation the ETGraph, i.e., eye-tracking graph. Visual282 izing such a graph can be achieved using force-directed graph ${ }_{283}$ layout algorithms or projection-based methods such as multi284 dimensional scaling. In the original page view, fixations and 285 saccades can be plotted to produce scanpaths. However, nodes 286 are solely constrained by their spatial locations on the page. ${ }_{287}$ With this stringent constraint, large saccadic amplitudes may 288 not always be of interest (e.g., a saccade moves from the end 289 of one line to the beginning of the next line). Unlike the page 290 view, nodes in the graph view are not constrained by their cor291 responding spatial locations of fixation clusters and the graph 292 structure are dictated by node connectivity (i.e., the actual read${ }_{293} \mathrm{ing}$ ). Therefore, the graph view can reveal the underlying nature 294 of the reading pattern.

295 Finally, we design ETGraph so that users can smoothly tran296 sit between SPSP, SPMP, and MPSP. This facilitates the exam${ }_{297}$ ination of reading patterns from both global and local perspec- 


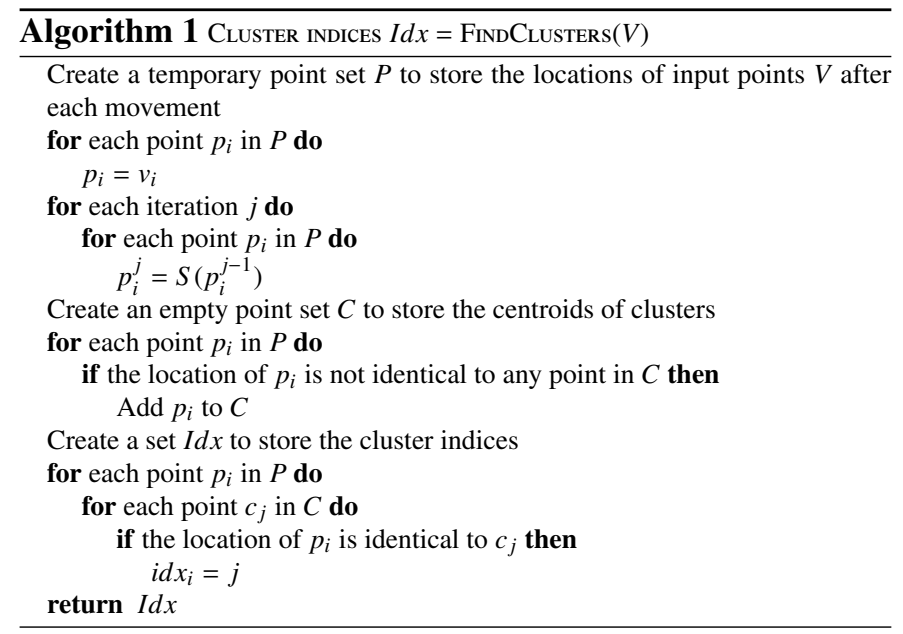

298 tives while making connections between them.

\section{4.1. Mean-Shift Algorithm}

300 We use the mean-shift algorithm, a density-based clustering 301 to group fixations. This algorithm is deterministic and robust to 302 outliers. In addition, it does not require users to input the num303 ber of clusters. Santella and DeCarlo [18] also demonstrated 304 the mean-shift method produces better quality of clusters com305 pared to the k-means clustering or expectation maximization 306 (EM) algorithms. In the mean-shift algorithm, the input points 307 are moved to a denser configuration so that they are naturally ${ }_{308}$ grouped into clusters. Moving each point $p$ to a new location is 309 based on the locations of its neighbors

$$
S(p)=\frac{\sum_{j} \operatorname{ker}\left(p-p_{j}\right) p_{j}}{\sum_{j} \operatorname{ker}\left(p-p_{j}\right)},
$$

310 where the kernel function estimates the contribution of each 311 neighbor $p_{j}$, i.e.,

$$
\operatorname{ker}(d)=\exp \left(\frac{-d_{x}{ }^{2}-d_{y}{ }^{2}}{\epsilon^{2}}\right),
$$

312 where $d$ is the Euclidean distance between $p$ and $p_{j}$, and $d_{x}$ and ${ }_{313} d_{y}$ are the projections of $d$ along the $x$ and $y$ directions, respec${ }_{314}$ tively. $\epsilon$ is a given threshold and the points with a distance to ${ }_{315} p$ larger than $\epsilon$ are not considered. Since our data are collected 316 from text-reading experiments, the $x$ and $y$ directions are related 317 to word length and line spacing, respectively. We therefore re318 vise the kernel function to

$$
\operatorname{ker}(d)=\exp \left(\frac{-d_{x}^{2}}{\epsilon_{x}^{2}}\right) \times \exp \left(\frac{-d_{y}{ }^{2}}{\epsilon_{y}^{2}}\right),
$$

319 where $\epsilon_{x}$ is the average word length and $\epsilon_{y}$ is the line spacing. ${ }_{320}$ Algorithm 1 first moves the input points $V$ to a denser con${ }_{321}$ figuration $P$. This process stops when the number of iterations ${ }_{322}$ reaches a user-defined threshold or the locations of points in $P$ ${ }_{323}$ do not change. The points in $P$ that move to the same loca324 tion become a cluster. The locations are treated as the centroids 325 of clusters. We assign the points to clusters by measuring the ${ }_{326}$ distances between the points and the centroids.

\section{4.2. Transition Graph}

${ }_{328}$ After clustering all fixations of each page using the mean329 shift algorithm, we construct a transition graph. Each node in $3{ }_{3}$ the graph denotes a fixation cluster. A directed edge between ${ }_{331}$ two nodes represents a transition. A transition $i \rightarrow j$ occurs 332 between two clusters $i$ and $j$ if there is a saccade from a fixa${ }_{33}$ tion in $i$ to another fixation in $j$. The transition frequency $f_{i \rightarrow j}$ 334 is the number of transitions from $i$ to $j$. The directional transi335 tion probability $p_{i \rightarrow j}$ is the proportion between $f_{i \rightarrow j}$ and the total 336 number of transitions from $i$ to all the clusters (including itself). ${ }_{337}$ As such, a transition indicates a chance for one cluster to trans338 fer to another, and its probability measures how high the chance $3 з 9$ is. To draw the transition graph, we modify the Fruchterman${ }_{340}$ Reingold algorithm [27] by considering the transition probabil${ }_{341}$ ity when computing the attractive forces. Therefore, two nodes 342 with strong transitions are placed close to each other. However, ${ }_{343}$ the nodes may overlap with one another due to their sizes in 344 the drawing. To reduce the overlap while preserving the over345 all graph structure, we follow the layout adjustment solution ${ }_{346}$ given by $\mathrm{Gu}$ and Wang [28] which first triangulates the graph 347 and then applies four additional forces (bidirectional, unidirec348 tional, spring, and attractive forces).

\section{${ }_{349}$ 5. SPSP, SPMP, and MPSP}

ETGraph helps users identify common reading patterns and 351 outliers for analytical reasoning at three different levels of de352 tail: SPSP, SPMP, and MPSP. First, SPSP provides detailed ex353 amination of the reading patterns for one participant reading 354 one page. Second, extending single page to multiple pages, ${ }_{355}$ SPMP visualizes the reading patterns for one participant read356 ing continuous pages. This allows users to identify abnormal 357 behaviors across different pages which may, for instance, indi358 cate that the difficulty levels of some pages are different from 359 others. Third, extending single participant to multiple partici360 pants, MPSP aims to analyze the common reading pattern and ${ }_{361}$ different reading behaviors among participants.

\section{5.1. SPSP}

363 To help users better understand detailed reading behaviors 364 for SPSP, we provide several query functions, e.g., saccade out365 lier detection, MW highlighting, graph filtering, path anima366 tion, and repeated scanpath detection.

367 Saccade outlier detection automatically identifies the sac368 cade outliers that traverse a large distance (larger than a given 369 threshold) along the $x$ or $y$ direction. Users are allowed to 370 change the threshold. By default, the thresholds along $x$ and $y$ 371 directions are around $1 / 3$ of the page width and $1 / 4$ of the page 372 height, respectively. These saccade outliers indicate long eye 373 movements which may indicate abnormal reading patterns and ${ }_{374}$ possible MW. We further differentiate backward- and forward375 reading saccades. Backward-reading saccades indicate revisit376 ing earlier portions of the text while forward-reading saccades 377 may indicate foreshadowing. Figure 1 shows an example of 378 saccade outlier detection. Specifically, we show the informa379 tion of saccade outliers in each of the four views. 

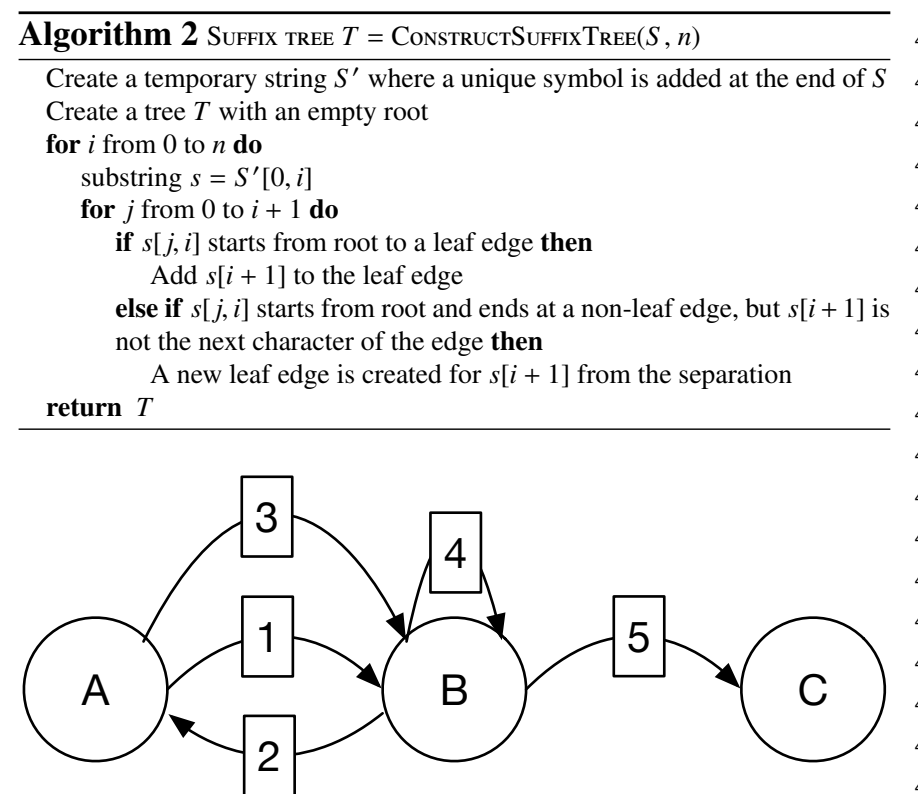

Figure 2: An example of the scanpath in the transition graph. The scanpath is $A B A B B C$, the corresponding string is $A B A B C$, and the repeated scanpath is $A B$.

MW highlighting visualizes the scanpath immediately be${ }_{381}$ fore and after instances of MW. As shown in Figure 1 (a) and 382 (b), the scanpath and its corresponding subgraph are highlighted звз in pink. This function provides a way for users to study MW in 384 the page, graph, and time views.

$385 \quad$ Graph filtering allows users to hide nodes (fixations) and 386 edges (saccades) that are not of interest. As users select one 387 or a group of nodes, we automatically hide the nodes that are 388 beyond a given distance to the selected node(s). The graph dis389 tance between two nodes is calculated using Dijkstra's algo$390 \mathrm{rithm}$. If two nodes that are not supposed to be connected in the 391 presumed reading order are linked in the graph view, this indi392 cates that at least one saccade outlier is present. Graph filtering 393 also allows users to filter the graph based on time information 394 and analyze the corresponding subgraph.

395 Path animation provides users the convenience of reviewing 396 scanpath animation. We identify the starting and ending fixa397 tions $f_{s}$ and $f_{e}$ for the animation. By default, they are the first 398 and last fixations on the page. However, users are allowed to se399 lect fixations from the page view or nodes from the graph view ${ }_{400}$ for $f_{s}$ and $f_{e}$. If the user selects two nodes from the graph view, ${ }_{401}$ we identify the first fixation in the first node as $f_{s}$ and the last ${ }_{402}$ fixation in the second node as $f_{e}$. If the two fixations selected ${ }_{403}$ do not follow the actual reading order, we swap $f_{s}$ and $f_{e}$. If 404 the user selects a node from the graph view and a fixation from 405 the page view, we ensure that $f_{s}$ occurs before $f_{e}$ in the actual ${ }_{406}$ reading order.

$407 \quad$ Repeated scanpath detection automatically detects repeated 408 scanpaths. We allow users to visualize them and find out their ${ }_{409}$ corresponding locations and time periods from the graph, page, 410 and time views. In addition, we allow users to play back the ${ }_{411}$ scanpaths to compare similarities and differences in scanpaths ${ }_{412}$ between different pages and different participants. In order to
${ }_{413}$ detect repeated scanpaths, we first convert the scanpath into a 414 string, and this string stores all the transitions between graph 415 nodes. Since we are more interested in transitions between ${ }_{416}$ nodes, we ignore all the transitions within the same node. Fig${ }_{417}$ ure 2 shows an example of the scanpath in the transition graph. ${ }_{418}$ We first assign IDs to the nodes. Therefore, the scanpath is ${ }_{419} A B A B B C$. Since we ignore all the self-transitions, the corre420 sponding string becomes $A B A B C$. By analyzing this string, we ${ }_{421}$ can detect interesting phenomenas, such as area revisitings (re${ }_{422}$ peated characters, $A$ and $B$ ), repeated scanpaths (repeated sub${ }_{423}$ strings, $A B$ ), and similar behaviors between two participants ${ }_{424}$ (common substrings of two given strings). To detect these phe425 nomena, we utilize the suffix tree [29] to identify the repeated ${ }_{426}$ substrings in a given string. The suffix tree is a tree structure ${ }_{427}$ that stores all the suffixes of a given string. Each edge repre${ }_{428}$ sents a substring. Therefore, all the non-leaf edges in the suffix ${ }_{429}$ tree represents repeated substrings. Constructing and searching 430 takes linear time which allows for efficient queries. We first add ${ }_{431}$ a unique symbol $\$$ at the ending of the given string to become ${ }_{432}$ a new string. This symbol is used to indicate the ending of the ${ }_{433}$ given string. At each iteration, we consider a substring starting ${ }_{434}$ from string indices 0 to $i$, where $i$ increases from 0 to $n-1$, and ${ }_{435} n$ is the length of the new string. Then, within each iteration, 436 all the suffixes of the substring are inserted into the suffix tree ${ }^{437}$ as shown in Algorithm 2. For example, given a string $A B A$, ${ }_{438} A$ is the repeated substring. Once symbol $\$$ is attached to the $439 \mathrm{end}$, the given string becomes $A B A \$$. According to Algorithm ${ }_{440} 2$, substrings $A \$, B A \$, A B A \$$ would be inserted into the suf${ }_{441}$ fix tree. Therefore, edge $A$ has children $\$$ and $B A \$$. Then $A$ is ${ }_{442}$ identified as a repeated substring. If no such a special symbol ${ }_{443}$ is attached at the end of the given string, edge $A$ would become 444 part of $A B A$ and thus could not be identified. To identify the 445 common substrings of two given strings, we further add an${ }_{446}$ other special symbol between the two given strings to indicate 447 the separation of the two strings and use the whole string as 448 an input for the suffix tree. For example, given two strings $B A$ 449 and $A A$, without symbols separating them, the combined string ${ }_{450}$ would be $B A A A$. In this case, $A A$ will be considered as a sub451 string that repeats twice. However, it is not the case. If we add 452 a special symbol *, then the combined string would be $B A^{*} A A$. ${ }_{453}$ In this case, $A A$ only appears once as a substring. Note that the 454 two symbols ( $\$$ and $*$ ) are different. Using the ending symbol 455 for the separation of the two strings may lead to the missing of 456 the repeated substrings. To allow users to focus on prominent ${ }_{457}$ substrings, we removed the substrings which are substrings of 458 others, or less than a given length.

\section{5.2. $S P M P$}

460 To understand and compare different behaviors on different ${ }_{461}$ pages (SPMP), we generate a SPMP-supergraph that displays 462 the transition graphs of all pages. An example is shown in Fig463 ure 3. The transition graphs are arranged clockwise in a spi${ }_{464}$ ral shape. To reduce edge crossing between pages, we first fix 465 the positions of the first and last nodes at the middle-left and 466 middle-right parts of each subgraph, respectively. Then we ro${ }_{467}$ tate each subgraph one by one to reduce the length of the edge 468 connecting the adjacent pages. 


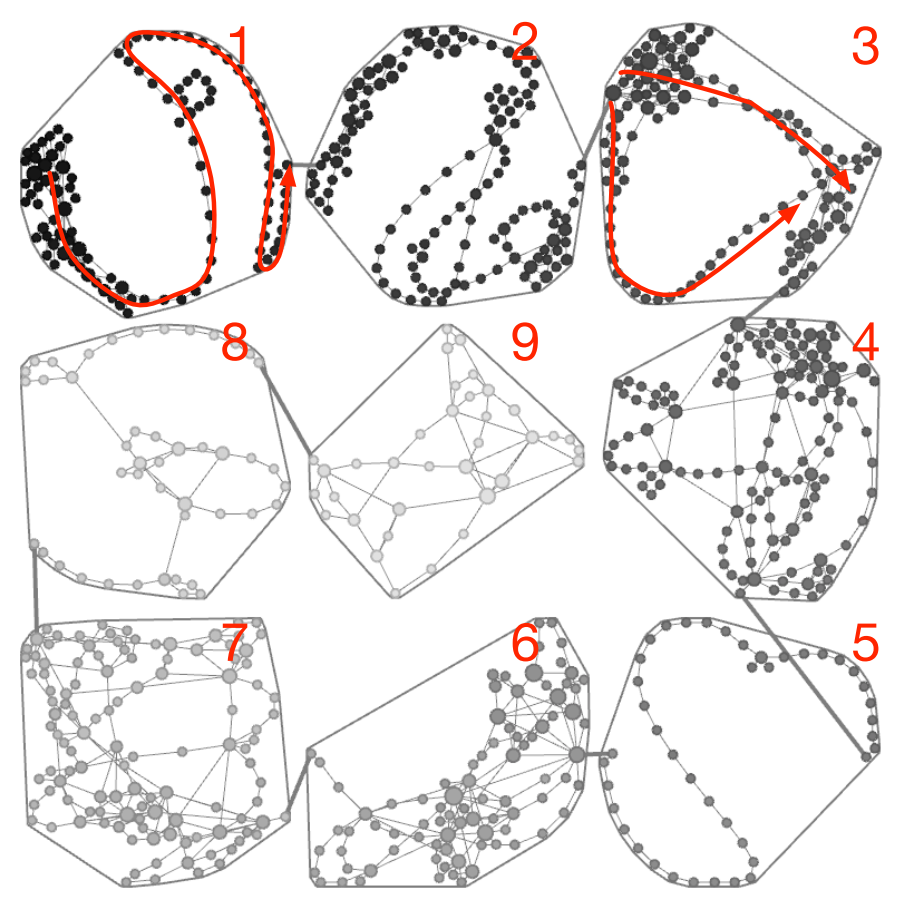

Figure 3: The SPMP-supergraph. Each subgraph corresponds to the transition graph of each page. The subgraphs are marked with their page numbers and are placed in a spiral along the clockwise order. In addition, we rotate each subgraph to reduce the length of the edge that connects two adjacent pages. The shading of nodes (from dark to light) indicates the presumed reading order.

\section{5.3. MPSP}

470 For MPSP, we cluster fixations on each page with all the 471 fixations of all participants on that page. After clustering, we 472 construct a MPSP-supergraph consisting of all the clusters of all ${ }_{473}$ participants of that page. As a result, edges with high frequen474 cies form the major reading trend of all participants. ETGraph 475 encodes higher frequency edges with darker gray colors so that 476 users can easily understand the overall reading patterns. How477 ever, it is difficult to notice the trend of saccade outliers since 478 those edges are usually drawn with lighter gray colors due to 479 their low frequencies. To highlight saccade outliers and iden480 tify their trend, we apply the edge bundling technique [30] to ${ }_{481}$ bundle saccade outliers as shown in Figure 4.

482 It is also important to cluster and compare participants in 483 order to analyze their similarities and differences. We provide ${ }_{484}$ two approaches. First, we utilize scarf plots and histograms in 485 the time and statistics views to show explicit information (e.g., 486 section length in milliseconds, saccade outlier distribution) for ${ }_{487}$ comparison. Second, we calculate the similarities between par488 ticipants based on the graph information. For MPSP, the transi${ }_{489}$ tion graph of a participant for a page is a subgraph of the MPSP490 supergraph. Therefore, by comparing the similarities between 491 two subgraphs, we can calculate the similarities between these 492 two participants. The difference between two participants can ${ }_{493}$ also be calculated based on their fixation distributions, repeated 494 scanpaths, etc. For each type of difference, we construct a dis495 tance matrix. We then normalize each distance matrix and add 496 them together to form the final distance matrix. Finally, we uti497 lize the k-means algorithm to cluster participants. The number

\begin{tabular}{|c|c|c|c|c|c|}
\hline & $\begin{array}{c}\text { mean } \\
\text { shift }\end{array}$ & $\begin{array}{c}\text { layout } \\
\text { generation }\end{array}$ & $\begin{array}{c}\text { layout } \\
\text { adjustment }\end{array}$ & $\begin{array}{c}\text { repeated } \\
\text { scanpath }\end{array}$ & $\begin{array}{c}\text { edge } \\
\text { bundling }\end{array}$ \\
\hline $\begin{array}{c}\text { single } \\
\text { participant (SP) }\end{array}$ & 1.283 & 18.941 & 3.968 & 0.143 & - \\
\hline $\begin{array}{c}\text { multiple } \\
\text { participants (MP) }\end{array}$ & 28.433 & 0.951 & 0.244 & - & 1.193 \\
\hline
\end{tabular}

Table 2: The timing results. The timing (in seconds) of SP is the total time for all pages of all participants. The timing (in seconds) of MP is the total time for all pages.

498 of clusters $n_{c}$ is chosen as $n_{c}=\sqrt{N / 2}$, where $N$ is the number 499 of participants. Based on the clustering, we allow users to se500 lect two participants for comparison as shown in Figures 8 and 5019.

\section{${ }_{502}$ 6. Results}

503 In the section, we first describe the data set and report the 504 timing performance. Then we demonstrate the results for SPSP, 505 SPMP, and MPSP, as well as the benefits and knowledge gained 506 using ETGraph. For the 10 research questions $(\mathbf{Q 1} \sim \mathbf{Q 1 0})$ 507 grouped into six categories $(\mathbf{C 1} \sim \mathbf{C 6})$, we add a note such as ${ }_{508}$ (C1-Q1) to show the category and the question that each result 509 answers.

\section{6.1. Data Set and Timing Performance}

511 The data set was generated from eye-gaze data collected 512 while participants read an excerpt from a book entitled "Soap513 bubble and the Forces which Mould Them" [31]. This text was 514 chosen as it was on a novel topic which would be relatively un515 familiar to a majority of readers. Eye-gaze data was collected 516 with a Tobii TX300 remote eye tracker with the sampling fre517 quency of $300 \mathrm{~Hz}$. The eye tracker was affixed below a monitor 518 set to a resolution of $1920 \times 1080$, which displayed the text. The 519 excerpt consisted of text from the first 35 pages of the book and 520 contained around 5700 words across 10 pages. Each partici${ }_{521}$ pant read the text for 20 minutes, and not every one was able 522 to finish reading all pages. Few participants read the final page ${ }_{523}$ (Page 10), so it has not been included in our analysis. Eye${ }_{524}$ gaze data were collected from both eyes and the data from each 525 eye were filtered and averaged together prior to eye-movement 526 detection. The data were then converted into a series of fixa527 tions using a dispersion-based filter. Using the Open Gaze And ${ }_{528}$ Mouse Analyzer (OGAMA) [32], the filter was set to detect fix529 ations if there were consecutive gaze points within a range of 53057 pixels (approximately 1 degree of visual angle) for longer 531 than $100 \mathrm{~ms}$, which is the shortest duration for naturalistic eye532 movements during reading $[11,33]$. Saccades were then calcu${ }_{533}$ lated from the fixations. The timing was collected on a PC with 534 an Intel 3.6 GHz CPU and 32 GB memory. The processed data ${ }_{535}$ set consists of 27 participants and 9 pages. The timing results ${ }_{536}$ are shown in Table 2.

${ }_{537}$ 6.2. $S P S P$

${ }_{538}$ Figure 5 shows an example of SPSP. In (a), each dot in ${ }_{539}$ the page view represents a fixation and the fixation clusters 540 are highlighted using convex hulls. In (b), each node in the ${ }_{541}$ graph view represents a fixation cluster and an edge represents 542 a transition between two clusters. In the graph, the nodes with 


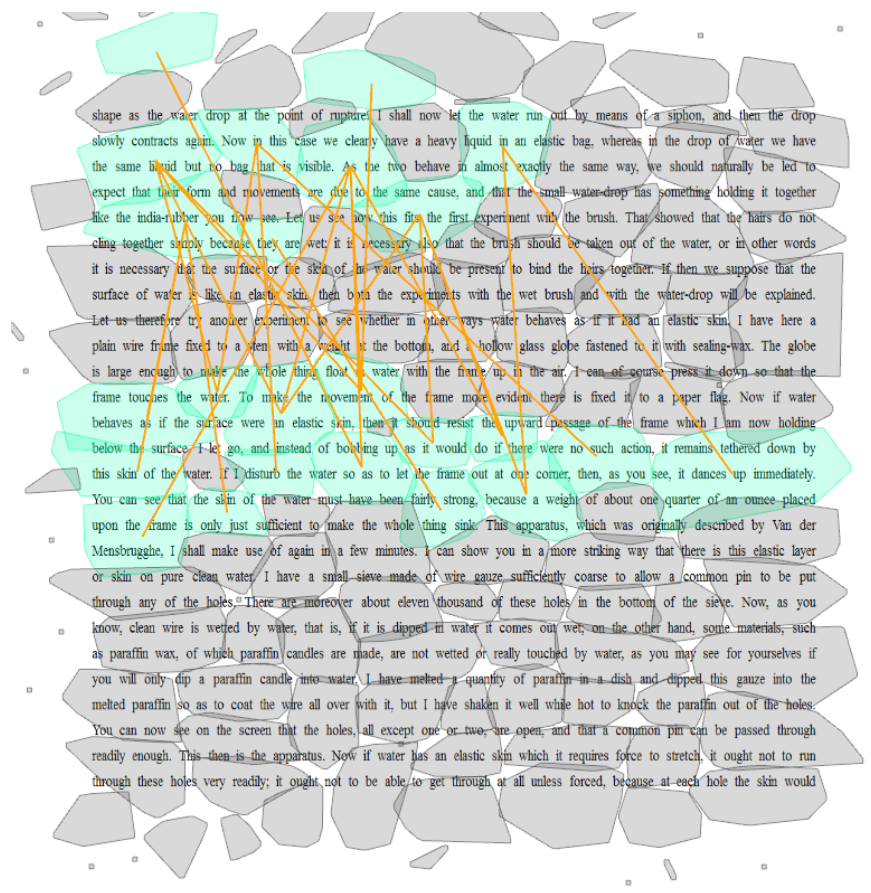

(a)

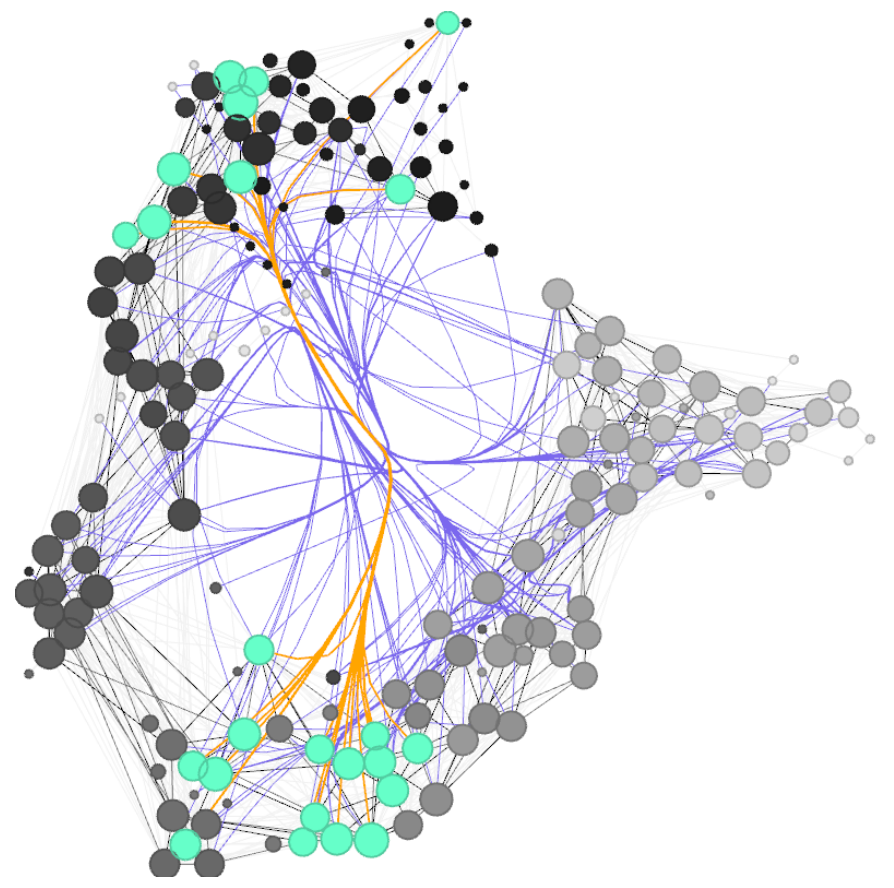

(b)

Figure 4: Edge bundling for MPSP. (a) The saccades of the selected bundles illustrate a close relationship between the two green areas in the page view. (b) The bundled edges are highlighted in blue while user-selected edge bundles are shown in orange. The regular (non-outlier) edges are shown in gray with higher frequency edges shown in darker gray.

${ }_{543}$ stronger transitions are placed closer to each other. The graph 544 view provides an overview of the reading pattern for a single ${ }_{545}$ page. The darkness of nodes (from dark to light) indicates the ${ }_{546}$ presumed reading order. Therefore, in general, nodes with sim547 ilar darkness values are placed nearby. We can observe that 548 the nodes in (b) are clearly separated into two groups. The 549 nodes in one group are in green and their corresponding fixa550 tions are located in the lower portion of the page as shown in 551 (a). The separation between these two groups of nodes could 552 indicate that this participant read the portion of text at the top 553 of the page separately from the portion of text at the bottom of 554 the page. Although some saccade outliers connect the top and 555 bottom portions of the page, more connections exist within the ${ }_{556}$ two portions. This indicates that there are stronger connections 557 within each portion than between the two portions. Of partic558 ular interest are the nodes in (b) that are not selected but are 559 connected to nodes that are. The corresponding fixations are 560 located in the first few lines of the page, which could indicate ${ }_{561}$ that the participant always returned to this location of the page 562 to reread (C1-Q1).

563 To understand the scanpath structures, besides regular read564 ing patterns, it is important to analyze saccade outliers. They 565 could indicate a portion of text that is difficult to understand or 566 a rereading pattern. We show an example in Figure 1. In (a) 567 and (b), the fixation clusters and nodes of the saccade outliers 568 are in yellow. The selected clusters and nodes are in green. Red 569 and blue edges indicate backward and forward saccade outliers, 570 respectively. Most of the saccade outliers in (a) are either tar571 geted at or moving from the upper portion of text. This could
572 indicate that the participant reread this portion of text. In ad573 dition, there is a saccade outlier from the middle of the page 574 toward the bottom of the page (outside of the screen), but the 575 connected nodes of the saccade outlier are close in (b), which 576 demonstrates that ETGraph gathers the nodes based on their 577 transition relations instead of their spatial closeness. In the time 578 view of (c), the vertical lines indicates the time slots where sac579 cade outliers occurred. The saccade outliers are displayed in red 580 and blue. (d) shows the distribution of the saccade outliers in 581 each of the three sections of the page. This distribution shows 582 that this participant had a large number of saccade outliers in ${ }_{583}$ the first section of the page $(\mathbf{C 2}-\mathbf{Q 3})$.

584 6.3. SPMP

585 An analysis of the reading patterns of a participant for mul${ }_{586}$ tiple pages can be done by studying the SPMP-supergraph of 587 the chosen pages along with their statistical information. Fig588 ure 3 displays a SPMP-supergraph of nine pages for a single 589 participant. The transition graphs of Pages 1, 5 and 8 are quite 590 simple since each transition graph forms a smooth curve. The 591 graph of Page 3 is very interesting because it consists of two 592 paths from the beginning to the end, which could mean that this ${ }_{593}$ participant read the page twice. However, this graph structure is 594 still simple compared to the graphs for Pages 4, 6 and 7. These 595 graphs consist of complex relationships between nodes which 596 could indicate that the participant read these pages backward 597 and forward many times (C1-Q6). Shifting to the correspond598 ing SPSP view allows for a more detailed examination of these 599 pages, which could offer additional insights. 


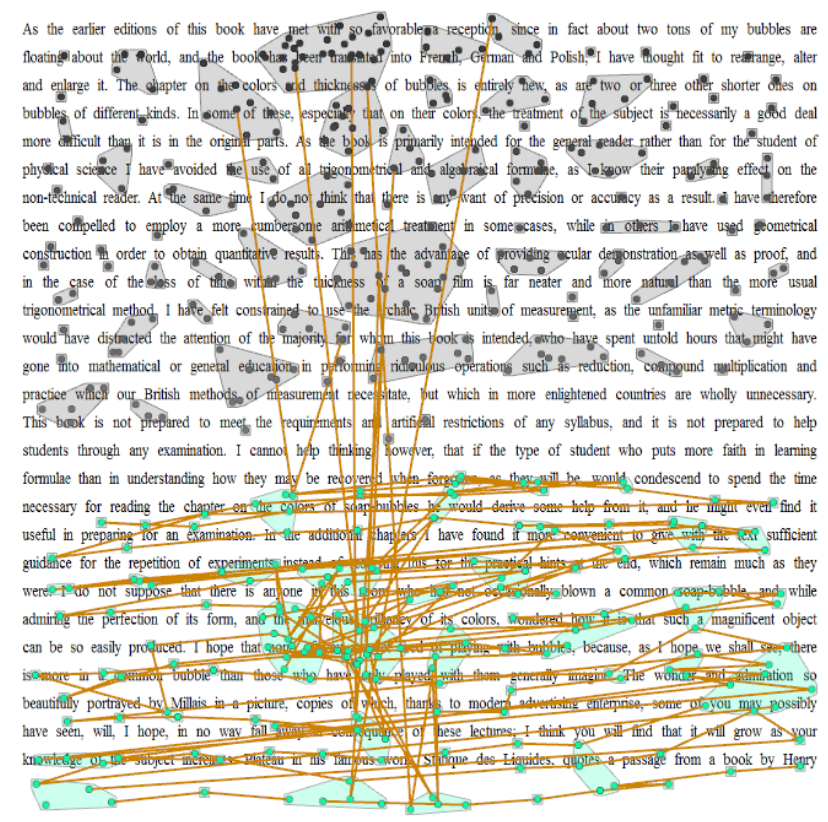

(a)

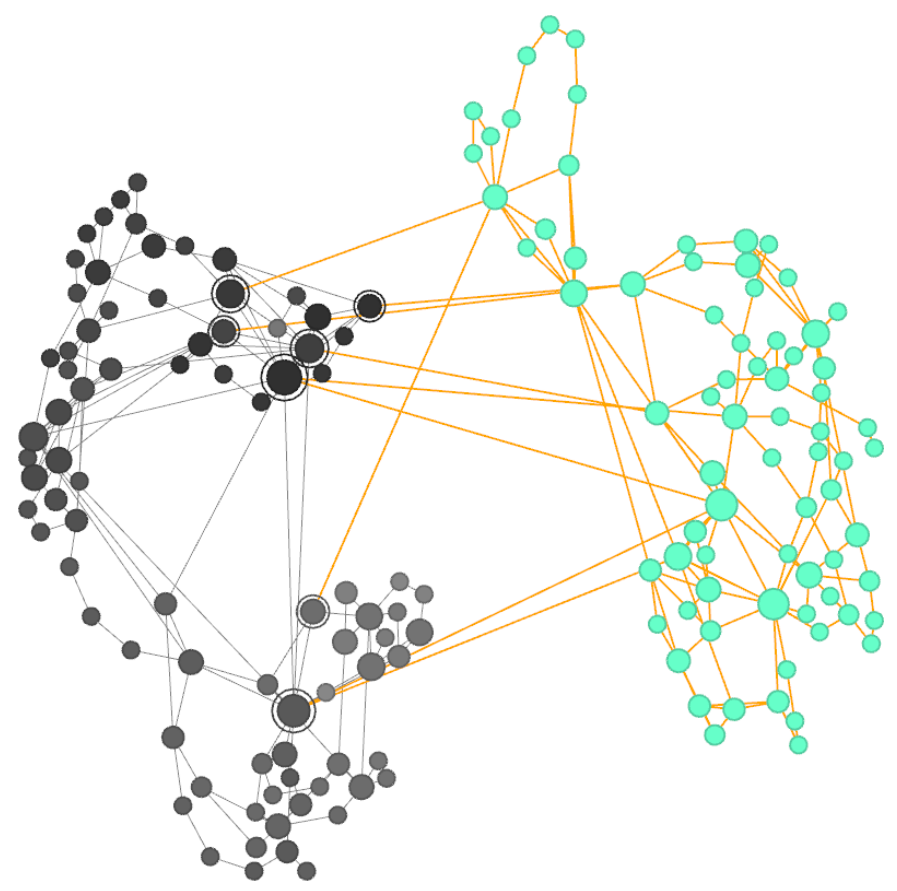

(b)

Figure 5: The transition graph of SPSP shows the clear separation of two parts in reading by the participant. An interesting repeated rereading pattern is also identified in green. The lower portion of the text in (a) corresponds to the selected nodes in (b).

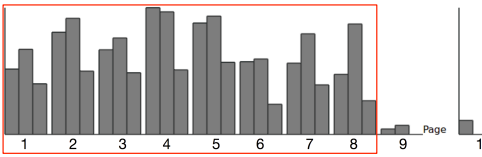

(a)

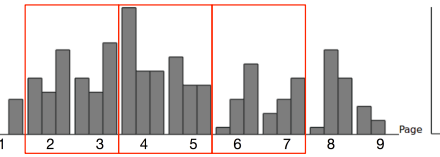

(b)

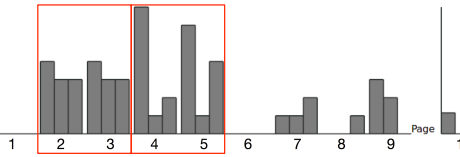

(c)

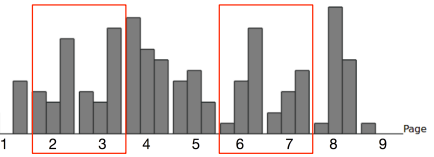

(d)

Figure 6: Frequency distributions of (a) fixations, (b) saccade outliers, (c) saccade outliers with large $y$ distances, and (d) saccade outliers with large $x$ distances.

600 SPMP also allows a comparison of pages by displaying sta601 tistical information for each page, which could indicate consis602 tency of reading patterns across all pages. The charts in Fig${ }_{603}$ ure 6 show the distribution of fixations (a), saccade outliers (b), 604 saccade outliers with large $y$ distances (c), and saccade outliers 605 with large $x$ distances (d). In (a), the fixation distributions are 606 quite similar among the first eight pages, which indicates sim607 ilar reading patterns. In (b), (c) and (d), the saccade outlier 608 distributions are similar between Page 2 and Page 3. In (b) and 609 (c), the saccade outlier distributions are similar between Page ${ }_{610} 4$ and Page 5. In (b) and (d), the saccade outlier distributions 611 are similar between Page 6 and Page 7. We conclude that there 612 were similar reading patterns between these pages, especially 613 adjacent pages (C6-Q7).

614 Besides showing the structures of the scanpaths, ETGraph 615 may be useful to find the patterns of MWs. Figure 7 shows ${ }_{616}$ MWs of a participant. In (a), the pink nodes indicate the ap${ }_{617}$ pearance of MWs. (b) and (c) show the page and graph views 618 of Page 2, respectively, (d) and (e) show the page and graph 619 views of Page 5, respectively. In (b) and (d), the scanpaths 620 around the MWs consist of saccade outliers. So, in (c) and (e), ${ }_{621}$ the subgraphs around the MWs span a large area. We can see 622 that ETGraph could provide a hint for users to detect pages con${ }_{623}$ sisting of MWs. If there is more than one episode of MW per
624 page, the subgraphs of MWs may consist of saccade outliers 625 and have overlap between them (C4-Q5).

\section{6.4. MPSP}

${ }_{627}$ To show the overall reading patterns of MPSP, we bundle 628 the edges to observe their trends, as shown in Figure 4. In (b), ${ }_{629}$ only the saccade outliers are bundled since bundling all edges 630 would hide the trend in the saccade outliers. The regular (non${ }_{631}$ outlier) edges are shown in various gray colors and the darkness ${ }_{632}$ of each edge shows its transition frequency. These gray edges ${ }_{633}$ give an impression of common reading patterns (C1-Q8). In ${ }_{634}$ contrast, the saccade outliers are bundled and highlighted in 635 blue. Nodes or bundles can be selected for observing their cor${ }_{636}$ responding fixations and saccades. The selected bundles are ${ }_{637}$ shown in orange and their connected nodes are shown in green. ${ }_{638}$ The corresponding page view in (a) illustrates a strong relation639 ship between the areas highlighted in green.

Besides showing the overall patterns of all participants, we ${ }_{641}$ can also cluster participants based on their attributes, e.g., fix642 ation distribution, graph similarities, and repeated scanpaths ${ }_{643}$ (C5-Q10). Figure 8 shows a comparison of two participants 644 who are in the same cluster based on fixation distribution. In 645 (b), blue nodes belong to one participant and red nodes belong ${ }_{646}$ to another, while gray nodes belong to both of them. The dark 

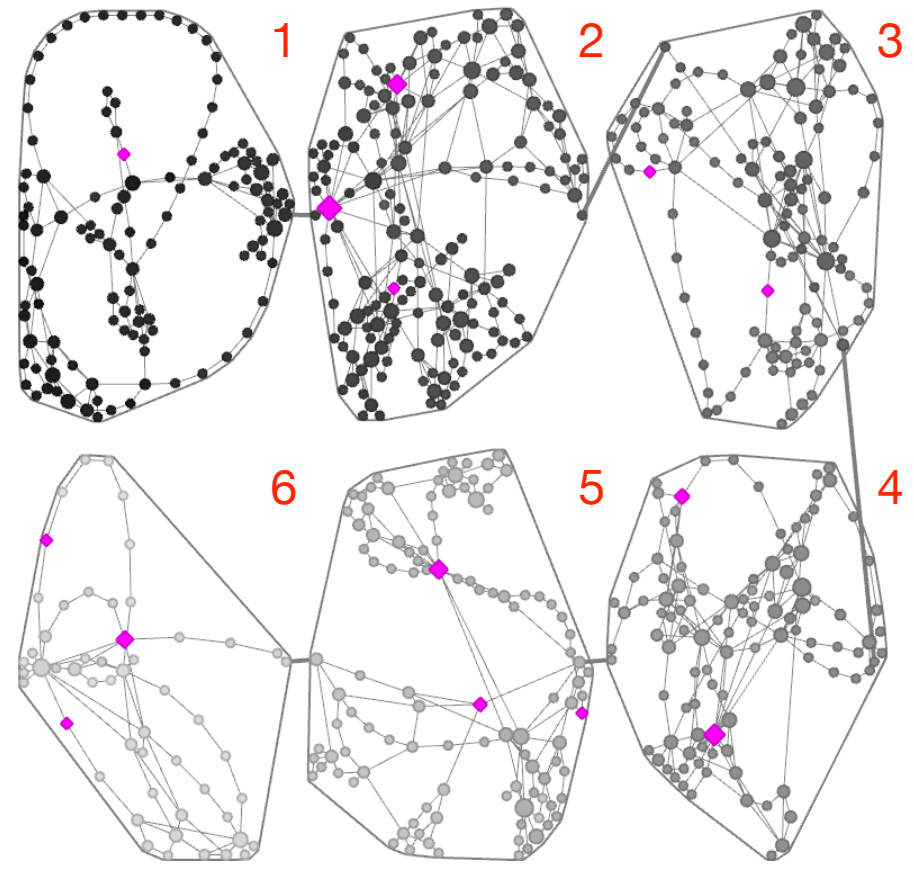

(a)

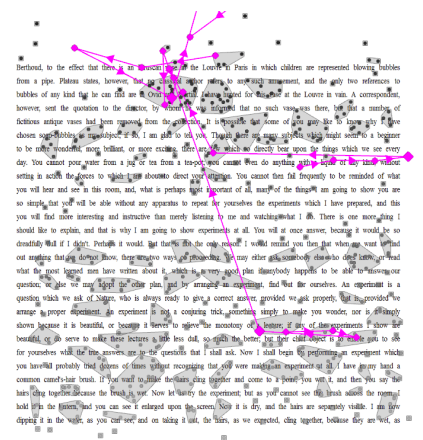

(b)

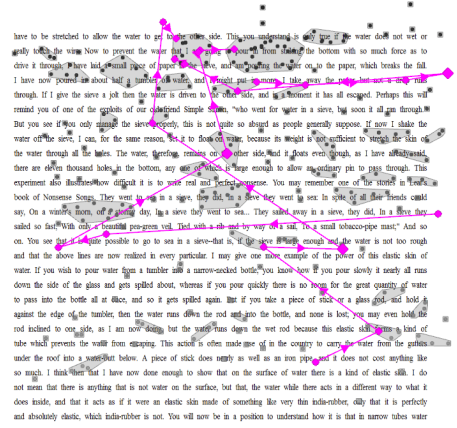

(d)

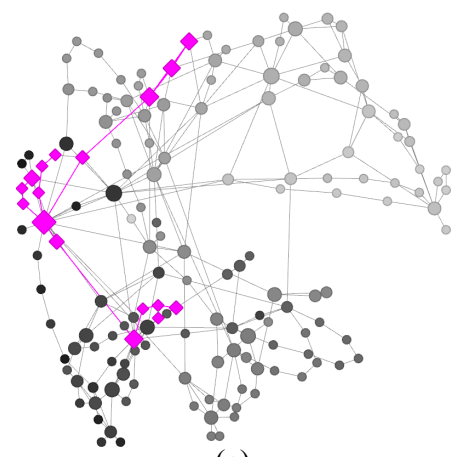

(c)

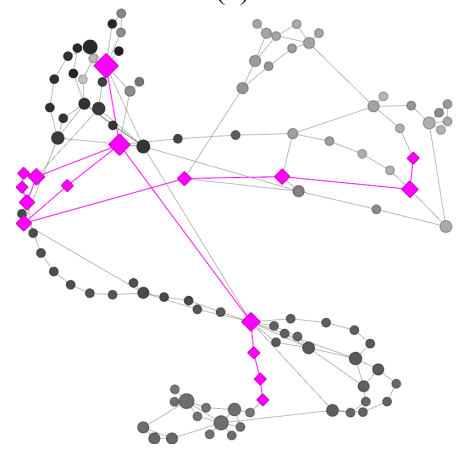

(e)

Figure 7: Visualization of MW of a participant for SPMP. In (a), the pink nodes indicate the occurrence of MW. (b) and (c) are the page and graph views of Page 2, respectively. (d) and (e) are the page and graph views of Page 5, respectively.

${ }_{647}$ edges belong to both participants and the gray ones do not. In $677 y$ distances do not occur in any repeated scanpaths for all par648 this example, most of the nodes and a large number of edges 678 ticipants. This indicates that the participants did not revisit two 649 are shared, which indicates that their corresponding graphs are 679 portions of text that have a large $y$ distance. However, it is not 650 also quite similar. From (a), we can see that the two partic- 680 the case for saccade outliers with large $x$ distances. Figure 10 651 ipants share quite a few fixation clusters shown in gray. The 681 (a) shows the repeated scanpaths and saccade outliers with large ${ }_{652}$ clusters that differ are located at the boundaries of the page, $682 x$ distances. The repeated scanpaths are shown in gray while the ${ }_{653}$ shown in blue and red. In addition, they both have a relative 683 saccade outliers are highlighted in red and blue bars indicating 654 lack of fixations in the middle area, highlighted in yellow. (c) 684 backward and forward saccade outliers, respectively. Repeated 655 displays the time of saccade outliers in the gray time views at 685 scanpaths occur when a participant rereads some portion of text. 656 the top and bottom, and the shared repeated scanpaths in the 686 A repeated scanpath may represent a scan of the text or a return 657 middle time view. There are a large number of colored bars 687 to a particular sentence (C3-Q4). When a participant tries to 658 which shows that the two participants shared a large number of 688 understand a large paragraph and a repeated scanpath is only 659 repeated scanpaths. This further indicates that the two partic- 689 a part of it, then this repeated scanpath is only a scan. This 660 ipants had similar reading patterns. (d) compares the fixation 690 is different from when the time gap between the corresponding ${ }_{661}$ distributions of all pages and similar fixation distributions can 662 be observed (C6-Q2, C6-Q9).

691 scanpaths of a repeated scanpath is short and there is a sac692 cade outlier between them. In Figure 10, we can see from (a)

Figure 9 shows a comparison of two participants who are 693 that there are some repeated scanpaths overlapped with saccade 664 in two different clusters based on fixation distribution. In (b), 694 outliers with large $x$ distances highlighted in the red rectangle. 665 there are more blue nodes than red nodes. From (a), we can 695 These repeated scanpaths may be more interesting than others. 666 see that there is a large number of blue fixation clusters in the 696 In this figure, repeated scanpaths that consist of saccade out667 middle of the page and there are no red fixation clusters, which 697 liers are shown in the red rectangle. The corresponding time 668 indicates that the participant in red did not read those portions 698 view is shown in (b), and the corresponding selected repeated ${ }_{669}$ at all. In (c) there are only four colored bars which show that ${ }_{699}$ scanpaths are shown in (c), (d), and (e). The page view shows 670 the two participants only shared two repeated scanpaths. This 700 that this participant reread this sentence twice, ostensibly for 671 further indicates that the two participants had different reading 701 better understanding.

672 patterns. (d) compares the statistics of fixations of all the pages 673 and shows different distributions (C6-Q2, C6-Q9).

${ }_{674}$ We also provide statistical information to help users identify 675 similarities and differences between all participants. For exam$676 \mathrm{ple}$, in our data, we found that the saccade outliers with large

\section{7. User Study and Expert Evaluation}

To evaluate the effectiveness of ETGraph, we conducted a 704 user study and an expert evaluation. The user study mainly fo705 cused on the usefulness and usability of ETGraph, while the 


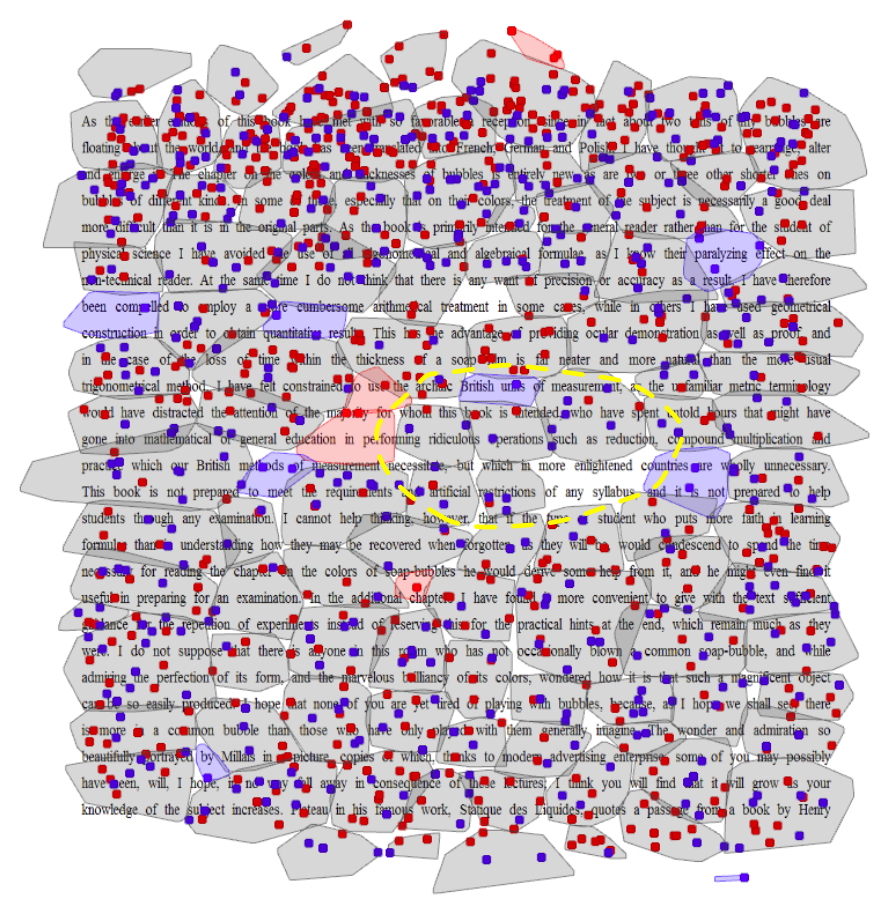

(a)

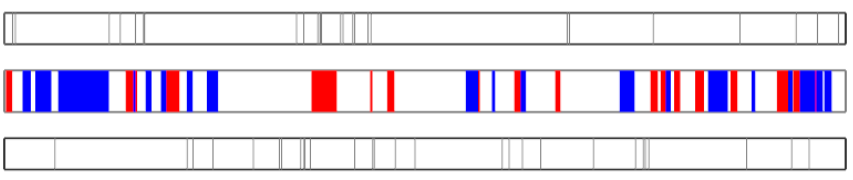

(c)

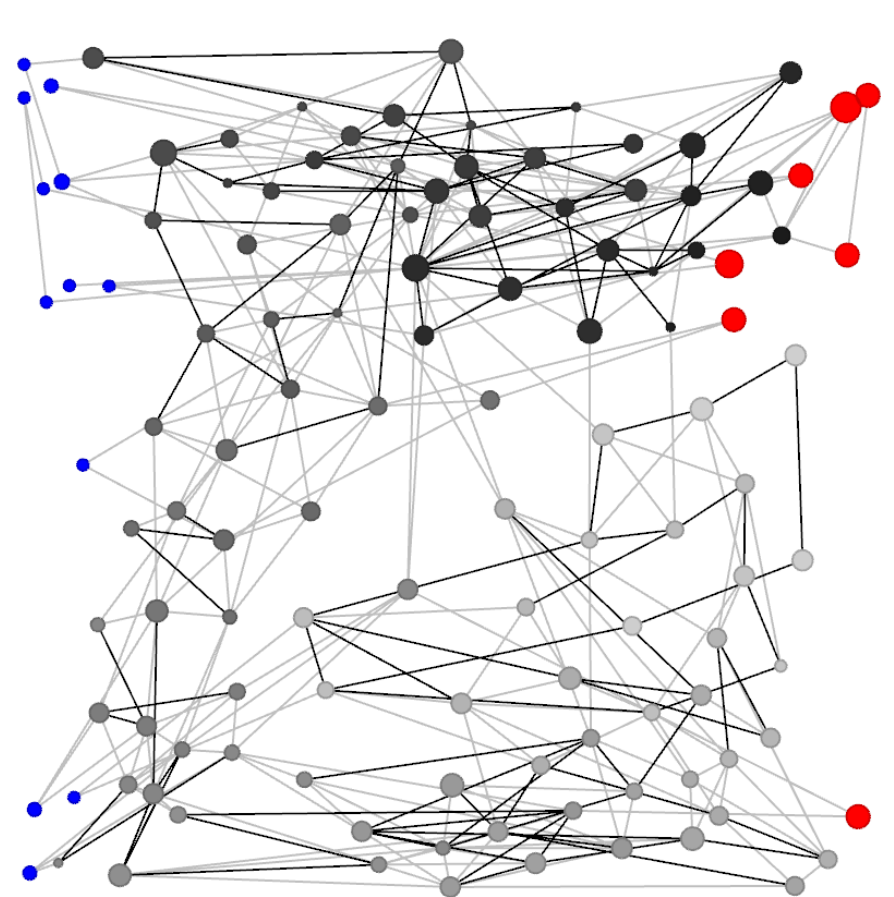

(b)

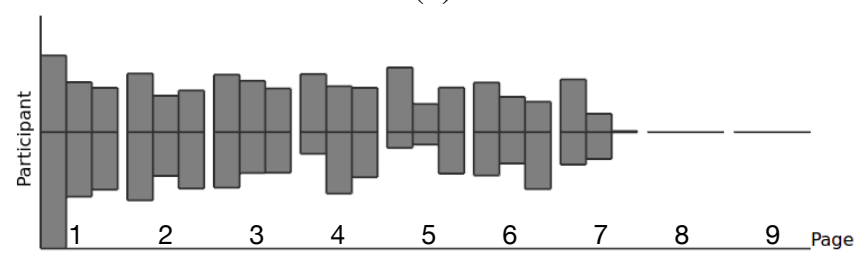

(d)

Figure 8: Comparison of two participants with similar fixation distributions. (a) The fixation distributions of the two participants. Note that both participants have few fixations in the middle area (highlighted in yellow). (b) Blue and red nodes belong to a single participant only and gray nodes belong to both participants. Dark edges belong to both participants. (c) Saccade outliers for the two participants are shown in the upper and bottom time views, while their shared repeated scanpaths are shown in the middle view. (d) A comparison of the statistics of fixations of the three sections of each page for the two participants. The participant with red nodes is at the top while the participant with blue nodes is at the bottom.

706 expert evaluation focused on the improvement and future direc707 tions.

\section{7.1. User Study}

709 We recruited five unpaid $\mathrm{PhD}$ students in our university to 710 evaluate the effectiveness of ETGraph. One student is from the 711 Department of Psychology and four students are from the De712 partment of Computer Science and Engineering. All five users 713 are analyzing MW in their respective $\mathrm{PhD}$ studies using differ714 ent kinds of data, e.g., physiology, facial features, or eye gaze. 715 The user study was conducted in a lab using the same PC for 716 each user. The users were first introduced to ETGraph and were 717 instructed about its design goals and main functions. Then they 718 were given ten minutes for free exploration to get familiar with 719 the system. After that, they were asked to complete six tasks 720 and a survey of seven general questions on the design of ET${ }_{721}$ Graph. These tasks were written on paper and the users hand 722 wrote their responses. The observer (i.e., one author of this 723 work) stood near by and took notes. After the study, he then 724 interviewed the users about their thoughts of the tasks and ET725 Graph.
Since ETGraph was mainly designed to visualize the read${ }_{727}$ ing patterns of the participants and help users identify MWs, 728 this user study focuses on evaluating the effectiveness of these 729 two aspects. As shown in Table 3, T1 T3 were designed 730 to evaluate the effectiveness in terms of revealing the reading ${ }_{731}$ patterns based on the graph representation, and T4 $\sim$ T6 were ${ }_{732}$ designed to evaluate the effectiveness in terms of helping the 733 users identify MWs.

In T1, the user was given three graphs. She was asked to 735 compare them based on the graph structures and identify the 736 one whose structure is different from those of the other two. ${ }_{737}$ Then the user was asked to observe the corresponding scan${ }_{738}$ paths and understand the relationships between graph structures 739 and scanpaths. Finally, she was asked to verify her observa740 tion through freely exploring the graphs and scanpaths. This ${ }_{741}$ task was designed to evaluate if the user was able to identify 742 the graph with an abnormal scanpath and infer why the graph 743 structures are different.

In T2, given a graph, the user was asked to circle node clus745 ters. Then she was asked to observe their correspondences in 


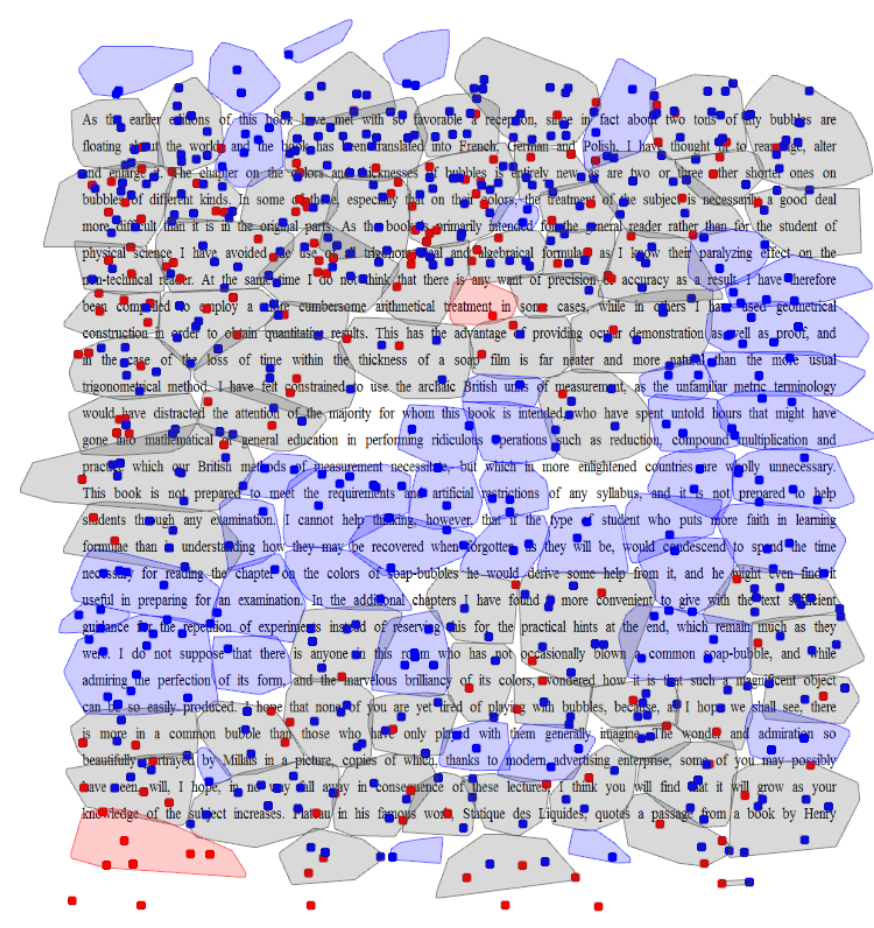

(a)

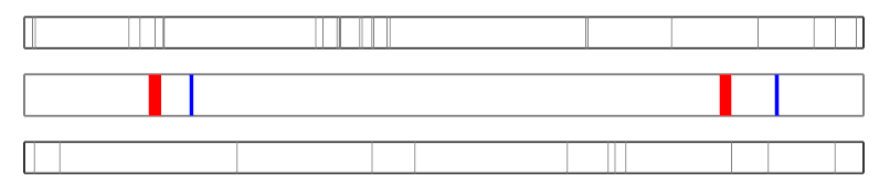

(c)

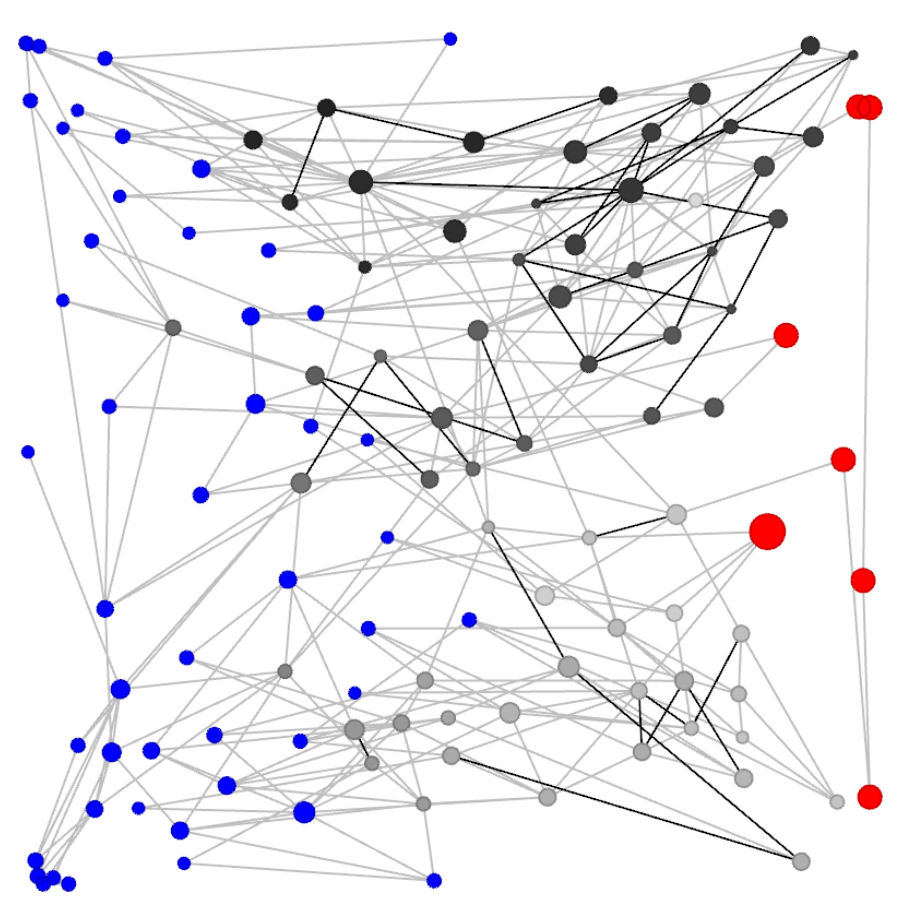

(b)

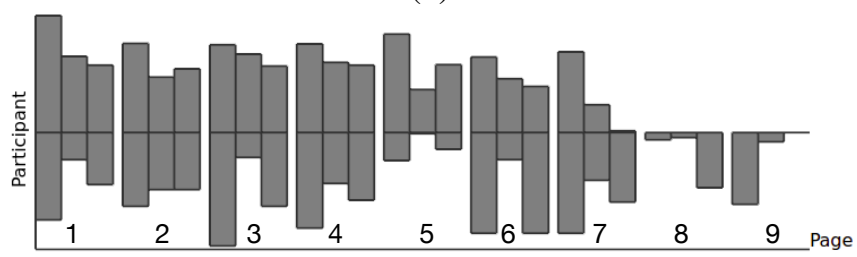

(d)

Figure 9: Comparison of two participants with different fixation distributions. (a) The fixation distributions of the two participants. Notice that the participant in red have no fixations in the middle area. (b) Blue and red nodes belong to a single participant only and gray nodes belong to both participants. Dark edges belong to both participants. (c) Saccade outliers for the two participants are shown in the upper and bottom time views, while their shared repeated scanpaths are shown in the middle view. (d) A comparison of the statistics of fixations of the three sections of each page for the two participants. The participant with red nodes is at the top while the participant with blue nodes is at the bottom.

746 the page view. Finally, she was allowed to explore the other 747 graphs and infer why some graphs had clusters while others did 748 not. This task was designed to evaluate if the user understood 749 that the nodes are placed nearby in the graph view due to their 750 strong relations in the scanpath.

751 In T3, given a graph, the user was asked to estimate which 752 edges in the graph represent saccade outliers. Then she was 753 asked to verify her guesses using ETGraph. Finally, she was 754 allowed to explore the other graphs to observe the correspon755 dence of outliers between the graph view and the page view. 756 This task was designed to evaluate if the user understood that 757 saccade outliers with large $y$ distances exist between two nodes 758 with either very different gray-scale colors or very long edges. ${ }_{759}$ The typical way for MW detection is to follow the anima760 tion of scanpath and identify MWs based on user experience. ${ }_{761}$ However, the scanpath could be long, dense, and self-occluded, 762 which makes it difficult for users to follow the animation, mem763 orize the animation history, and detect abnormal reading pat764 terns. ETGraph simplifies the scanpath during animation by ${ }_{765}$ preserving the most important features and reducing the user's
766 effort. Furthermore, since identifying MWs by watching the 767 animation requires a lot of domain knowledge, ETGraph sim768 plifies the process by helping users detect MWs through show769 ing a static view of the graph structures and visual hints for 770 saccade outliers. T4 $\sim$ T6 were designed to evaluate the effec771 tiveness of ETGraph in terms of helping identifying MWs. In 772 T4, the user was asked to watch the scanpath animation of sev773 eral participants reading different pages, and identify whether 774 MW was reported on each page. T5 and T6 asked the user to 775 identify whether MW was reported on the pages based on the 776 graph structures and saccade outliers, respectively.

777 The users could perform the tasks at their own pace. Each 778 session took about 30 to 60 minutes to complete. We summa779 rize the binary task completion scores for the six tasks in Ta780 ble 3 .

${ }_{781}$ We note that all users answered T1 and T2 correctly. For ${ }_{782} \mathbf{T 1}$, the users noticed that when a participant read from left to 783 right and from top to bottom, then the corresponding graph 784 presents a continuous transition from darker nodes to lighter 785 nodes, which conforms to the presumed reading order. How- 

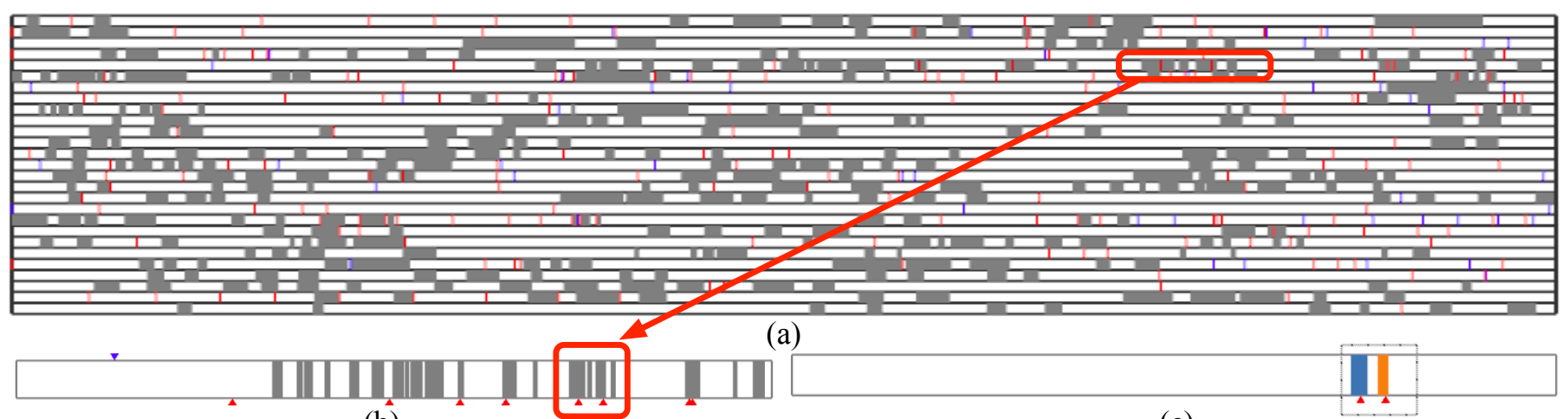

(b)

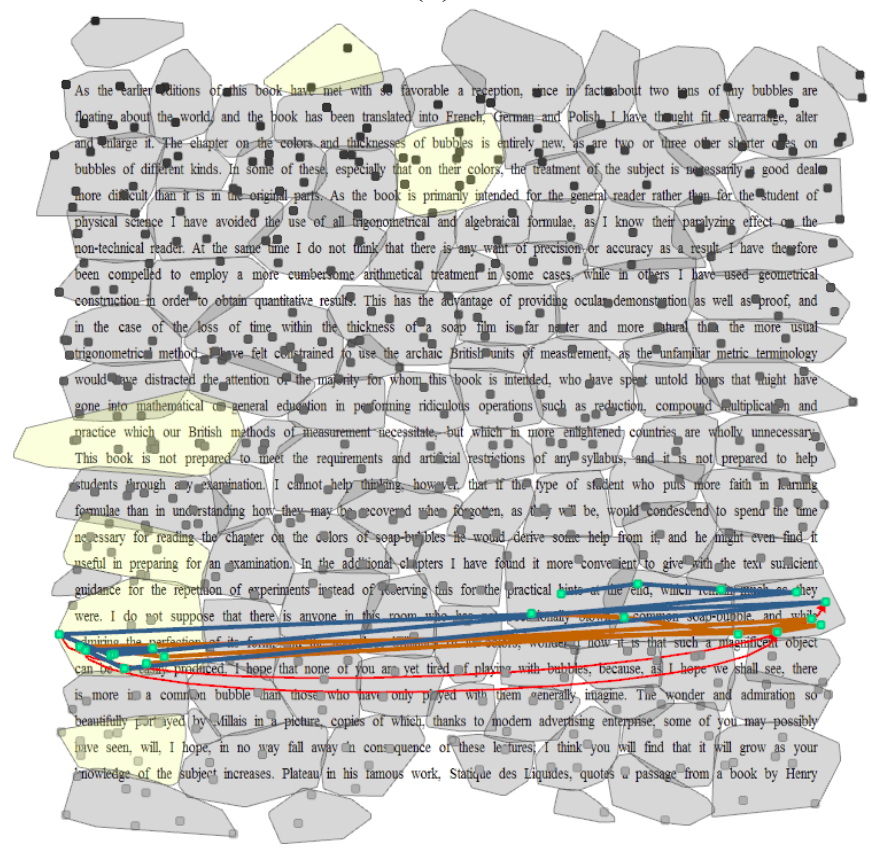

(d) (a)

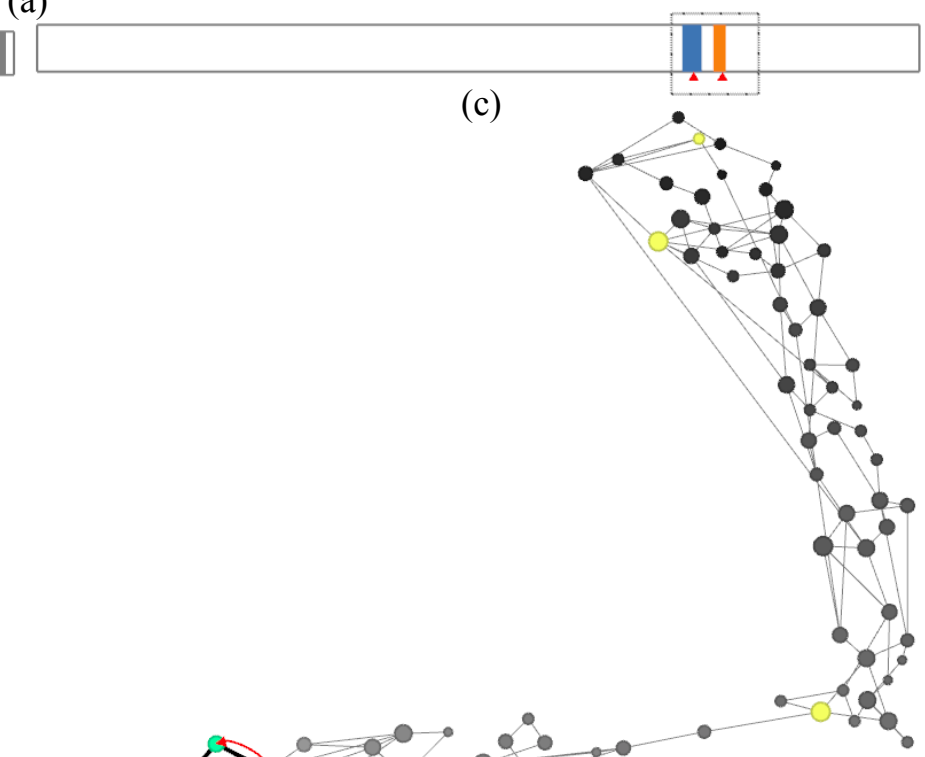

(e)

Figure 10: (a) The time view of saccade outliers and repeated scanpaths for MPSP, organized in a scarf plot. Repeated scanpaths are shown in gray while saccade outliers are highlighted in red and blue bars, indicating backward and forward saccade outliers, respectively. The red rectangle marks the repeated scanpaths that overlap with saccade outliers. (b) A participant is selected from (a) and the corresponding time view is shown. (c) The repeated scanpaths are selected. (d) and (e) are the corresponding page view and graph view for the selected repeated scanpaths, respectively.

786 ever, the graph structure became complex when there was a 803 out generation algorithm to users. This would help them better 787 large number of saccade outliers. For T2, the users drew circles 804 understand the graph so they would know that the edge repre788 to highlight the clusters in the graph, and selected each group 805 sents a saccade outlier when they observe such a phenomenon. 789 to verify their estimates. They concluded that if the participants 790 separated the text into portions and read each portion carefully, 791 then each portion would form a cluster in the graph.

For T4, the users tended to animate the entire scanpath to 807 understand the reading pattern and identify possible MW. How808 ever, it was sometimes difficult for them to do this because 792 For T3, the users could identify most of the saccade outliers 809 of visual clutter and the effort of remembering the previous 793 with large $y$ distances based on the edge lengths of the graph, 810 scanpath. To help users keep track of the reading pattern, ET794 but there was one saccade outlier that they all failed to identify. ${ }^{11}$ Graph only displays a few of the most currently displayed sac795 The edge of that saccade outlier linked two nodes that were 812 cades and all the previous saccade outliers during the anima796 close in the graph but one node was very dark and the other was 813 tion. The average score of successfully identifying MW was 0.7 797 very light. The fixation of the light node was very close to the ${ }_{814}$ for this task and most of the users considered this task difficult 798 bottom of the page and the dark node consisted of a lot of fixa- 815 to complete. All users except one made at least one incorrect 799 tions at the top of the page. Therefore, the dark node pulled the ${ }_{816}$ judgement. Most of the users stated that viewing an animation 800 light one close to itself and the corresponding edge length was 817 based on time that included saccade outliers helped them iden801 small. Failing to identify such an outlier shows that we should 818 tify rereading behaviors and abnormal reading patterns that may 802 have explained the details of ETGraph construction and the lay- ${ }_{819}$ include MW, but this function still requires them to have some 


\begin{tabular}{|c|c|c|c|}
\hline task & description & average score & standard deviation \\
\hline T1 & Identify the abnormal reading pattern based on the graph representations, and study their corresponding scanpaths. & 1 & 0 \\
\hline T2 & Circle the clusters in the graph and observe their corresponding portions in the page view. & 1 & 0 \\
\hline T3 & Circle the saccade outliers with large $y$ distances in the graph view and verify your guesses in the page view. & 0.8 & 0 \\
\hline T4 & Identity the pages with MW based on the animation of the whole scanpath. & 0.7 & 0.21 \\
\hline T5 & Identify the pages with MW based on the graph structures. & 0.8 & 0.27 \\
\hline T6 & Identify the pages with MW based on the saccade outliers in the graph view. & 0.8 & 0.45 \\
\hline
\end{tabular}

Table 3: The six tasks in the user study and user scores of the tasks.

${ }_{820}$ knowledge about typical reading patterns associated with MW. 821 For T5, the average score of successfully identifying MW 822 was 0.8 . All users stated that the graph structure could help de823 termine whether the participant read normally or was MW. This 824 task consisted of two subtasks. Three users who considered this 825 task easy completed it correctly. The remaining two users only 826 answered one small part of the task correctly. They both agreed ${ }_{827}$ that Figure 7 (c) (used in T5) showed a graph with a normal 828 structure. This result indicates that we need to train users about ${ }_{829}$ the graph structure in order to distinguish the differences be830 tween graphs with and without MW.

${ }_{831}$ For T6, the average score of successfully identifying MW ${ }_{832}$ was 0.8 . This task consisted of two subtasks. Four users who 833 considered this task easy completed it correctly. The remain834 ing user did not respond correctly. He studied the content con${ }_{835}$ nected by the saccade and decided that there was no MW present 836 if the content was related. However, this is not necessarily al${ }_{837}$ ways the case.

${ }_{838}$ On the general questions, all users agreed that ETGraph 839 was helpful for studying eye-tracking data. Based on the graph 840 structure, they could get an impression on whether the partici${ }_{841}$ pant followed a regular reading pattern or not. Saccade outlier 842 detection helped them identify saccade outliers and possible ${ }_{843}$ MW. Users also had some suggestions to improve ETGraph. ${ }_{844}$ Four of them suggested that we should provide more training 845 and explanation of ETGraph for better use. One even suggested ${ }_{846}$ that we list pages with or without MW, so that their differences 847 would be more obvious in ETGraph. Two users suggested that 848 we develop an iPad or Windows version for them to explore 849 further as our current system runs on Linux.

\section{7.2. Expert Evaluation}

${ }_{851}$ We also invited two domain experts: a professor and a $\mathrm{PhD}$ ${ }_{852}$ student whose research interest is identifying MW in eye-tracking ${ }_{853}$ data. We utilized the think-aloud protocol during the evalua${ }_{854}$ tion. The experts described their thoughts while completing the 855 tasks, and we summarized their comments after the evaluation. ${ }_{856}$ They both agreed that ETGraph is a very helpful tool for re857 searchers who are interested in studying eye movements.

858 The professor considered ETGraph a useful tool to identify 859 the reading patterns around MWs. He pointed out some sug860 gestions to improve ETGraph. First, he suggested that screen ${ }_{861}$ space should be added for the graph view so that users can se${ }_{862}$ lect multiple graphs for comparison. Second, he suggested that ${ }_{863}$ ETGraph should focus more on saccade outliers with large $y$ ${ }_{864}$ distances because they are important to identify rereading and ${ }_{865}$ MW. Saccade outliers with large $x$ distances might be due to ${ }_{866}$ the poor calibration of eye trackers and line jumps. For MPSP, ${ }_{867}$ he thought bundling outliers was helpful to identify the trend
868 of abnormal reading patterns. However, he suggested that we ${ }_{869}$ bundle the outliers separately for pages with or without MW. 870 This could help researchers study common patterns of MWs. 871 Finally, he thought that after identifying the common patterns 872 of the graphs with MW, using graph similarity measures could 873 help identify the pages with MW.

874 The student expert considered ETGraph to be a useful tool 875 because it provides a new approach to visualize eye-tracking 876 data. He has used tools like the Open Gaze and Mouse Analyzer 877 (OGAMA) [32] and has even written his own programs to ana878 lyze eye movements. These tools render the data using a scan879 path or heatmap. Visual clutter is inevitable when displaying a 880 scanpath, while heatmaps lack saccade information. ETGraph 881 addresses both limitations. In addition, he thought that ET${ }_{882}$ Graph helps to not only visualize eye movements but also gain 883 an understanding of patterns that are not obvious with other 884 tools. In addition, he is particularly interested in the repeated 885 scanpath detection function. For him, this function provides ad886 ditional information drawn from ETGraph besides saccade out${ }_{887}$ liers that could be used to detect MW. Finally, he thought that 888 our approach could help to engineer novel features for use in 889 machine learning based on observations drawn from ETGraph.

\section{8. Conclusions and Future Work}

891 We have presented ETGraph, a new approach that trans892 forms eye-tracking data of reading studies from the original ${ }^{893}$ page view to a graph-based representation. The graph view 894 presents fixation clusters as nodes and saccades as edges, and 895 it can reveal the very nature of the reading pattern by placing 896 nodes in the graph according to their connections, rather than 897 their fixed locations in the page. Through brushing and linking, 898 users are able to explore eye-tracking data from multiple per$g_{899}$ spectives. We demonstrate the usefulness of ETGraph by pre900 senting results generated from studying single participant sin901 gle page, single participant multiple pages, and multiple partic${ }_{902}$ ipants single page. The feedback from the domain experts and 903 a group of student researchers confirms the effectiveness of our 904 approach.

905 The current implementation of ETGraph has some limita906 tions. First, ETGraph helps users identify whether or not MWs 907 happen in pages, but it cannot tell accurately when and where 908 MWs happen. Second, MW detection in ETGraph may not be 909 extended to analyzing other types of data such as videos. MW 910 detection in our reading context assumes that the participants ${ }_{911}$ should follow a normal reading pattern (from left to right, from 912 top to bottom), and if they do not, MW may happen. Clearly, 913 this assumption does not hold anymore for other types of data. 
${ }_{914}$ Third, the clustering of participants is based on their graph sim915 ilarities, which does not allow users to manually select certain 916 graph attributes for more flexible participant classification.

${ }_{917}$ In the future, we would like to further apply graph min918 ing techniques, such as solutions for graph alignment or match${ }_{919}$ ing, to ETGraph, and investigate common reading patterns and 920 abnormal reading behaviors. The goal is to help users detect 921 a wide variety of cognitive and affective phenomena, such as 922 mind wandering, cognitive load, inference generation, and bore${ }_{923} \mathrm{dom}$, in a visually guided manner. We would also develop ${ }_{924}$ graph-based visual analytics tools for studying some other eye925 tracking data, such as data recorded for dynamic stimuli such 926 as videos.

\section{Acknowledgements}

${ }_{928}$ This research was supported in part by the U.S. National ${ }_{929}$ Science Foundation (NSF) through grants DRL-1235958, IIS930 1456763, IIS-1455886, and IIS-1523091. Any opinions, find931 ings and conclusions, or recommendations expressed in this pa${ }_{932}$ per are those of the authors and do not necessarily reflect the ${ }_{933}$ views of the NSF. The authors would like to thank the anony${ }_{934}$ mous reviewers for their insightful comments.

\section{${ }_{935}$ References}

[1] Smallwood J, Schooler JW. The restless mind. Psychological Bulletin 2006;132(6):946-58.

[2] Killingsworth MA, Gilbert DT. A wandering mind is an unhappy mind. Science 2010;330(6006):932-.

[3] Smallwood J, Fishman DJ, Schooler JW. Counting the cost of an absent mind: Mind wandering as an underrecognized influence on educational performance. Psychonomic Bulletin \& Review 2007;14(2):230-6.

[4] Kane MJ, McVay JC. What mind wandering reveals about executivecontrol abilities and failures. Current Directions in Psychological Science 2012;21(5):348-54

[5] Randall JG, Oswald FL, Beier ME. Mind-wandering, cognition, and performance: A theory-driven meta-analysis of attention regulation. Psychological Bulletin 2014;140(6):1411-31.

[6] Bixler R, D’Mello S. Toward fully automated person-independent detection of mind wandering. In: Proceedings of International Conference on User Modeling, Adaptation, and Personalization. 2014, p. 37-48.

[7] Reichle ED, Reineberg AE, Schooler JW. Eye movements during mindless reading. Psychological Science 2010;21(9):1300-10.

[8] Feng S, D'Mello S, Graesser A. Mindwandering while reading easy and difficult texts. Psychonomic Bulletin \& Review 2013;20(1):586-92.

[9] Smallwood J, Davies JB, Heim D, Finnigan F, Sudberry M, O'Connor $\mathrm{R}$, et al. Subjective experience and the attentional lapse: Task engagement and disengagement during sustained attention. Consciousness and Cognition 2004;13(4):657-90.

[10] Franklin MS, Broadway JM, Mrazek MD, Smallwood J, Schooler JW. Window to the wandering mind: Pupillometry of spontaneous thought while reading. The Quarterly Journal of Experimental Psychology 2013;66(12):2289-94

964 [11] Rayner K. Eye movements in reading and information processing: 20 years of research. Psychological Bulletin 1998;124(3):372-422.

966 [12] Franklin MS, Smallwood J, Schooler JW. Catching the mind in flight: 967 Using behavioral indices to detect mindless reading in real time. Psychonomic Bulletin \& Review 2011;18(5):992-7.

69 [13] Bixler R, D’Mello S. Toward fully automated person-independent detection of mind wandering. In: Proceedings of International Conference on User Modeling, Adaptation, and Personalization. 2014, p. 37-48.

72 [14] Raschke M, Blascheck T, Burch M. Visual analysis of eye tracking data In: Huang W, editor. Handbook of Human Centric Visualization. Springer New York; 2014, p. 391-409.
[15] Tory M, Atkins MS, Kirkpatrick AE, Nicolaou M, Yang GZ. Eyegaze analysis of displays with combined 2D and 3D views. In: Proceedings of IEEE Symposium on Information Visualization. 2005, p. 519-26.

[16] Duchowski AT. A breadth-first survey of eye tracking applications. Behavior Research Methods, Instruments, and Computers 2002;34(4):45570.

[17] Blascheck T, Kurzhals K, Raschke M, Burch M, Weiskopf D, Ertl T. State-of-the-art of visualization for eye tracking data. In: Eurographics Conference on Visualization - State-of-the-Art Reports. 2014, p. 83-2.

[18] Santella A, DeCarlo D. Robust clustering of eye movement recordings for quantification of visual interest. In: Proceedings of Symposium on Eye Tracking Research and Applications. 2004, p. 27-34.

[19] S̆pakov O, Räihä KJ. KiEV: A tool for visualization of reading and writing processes in translation of text. In: Proceedings of Symposium on Eye Tracking Research and Applications. 2008, p. 107-10.

[20] Goldberg JH, Helfman JI. Scanpath clustering and aggregation. In: Proceedings of Symposium on Eye Tracking Research and Applications. 2010, p. 227-34.

3 [21] Tang S, Ronan GR, Christian V. EyeMap: A software system for visualizing and analyzing eye movement data in reading. Behavior Research Methods 2012;44(2):420-38.

[22] Tsang HY, Tory M, Swindells C. eSeeTrack - visualizing sequential fixation patterns. IEEE Transactions on Visualization and Computer Graphics 2010;16(6):953-62.

[23] Burch M, Beck F, Raschke M, Blascheck T, Weiskopf D. A dynamic graph visualization perspective on eye movement data. In: Proceedings of Symposium on Eye Tracking Research and Applications. 2014, p. 151-8.

[24] Burch M, Kull A, Weiskopf D. AOI Rivers for visualizing dynamic eye gaze frequencies. Computer Graphics Forum 2013;32(3):281-90.

04 [25] Andrienko G, Andrienko N, Burch M, Weiskopf D. Visual analytics methodology for eye movement studies. IEEE Transactions on Visualization and Computer Graphics 2012;18(12):2889-98.

[26] Blascheck T, John M, Kurzhals K, Koch S, Ertl T. VA ${ }^{2}$ : A visual analytics approach for evaluating visual analytics applications. IEEE Transactions on Visualization and Computer Graphics 2016;22(1):61-70.

[27] Fruchterman TMJ, Reingold EM. Graph drawing by force-directed placement. Software - Practice and Experience 1991;21(11):1129-64.

[28] Gu Y, Wang C. TransGraph: Hierarchical exploration of transition relationships in time-varying volumetric data. IEEE Transactions on Visualization and Computer Graphics 2011;17(12):2015-24.

[29] Ukkonen E. On-line construction of suffix trees. Algorithmica 1995;14(3):249-60.

7 [30] Holten D, van Wijk JJ. Force-directed edge bundling for graph visualization. Computer Graphics Forum 2009;28(3):983-90.

1019 [31] Boys CV. Soap-Bubbles, and the Forces which Mould Them. Cornell University Library; 1890.

[32] Voßkühler A, Nordmeier V, Kuchinke L, Jacobs AM. OGAMA (open gaze and mouse analyzer): Open-source software designed to analyze eye and mouse movements in slideshow study designs. Behavior Research Methods 2008;40(4):1150-62.

[33] Holmqvist K, Nyström M, Andersson R, Dewhurst R, Jarodzka H, Van de Weijer J. Eye Tracking: A Comprehensive Guide to Methods and Measures. Oxford University Press; 2011. 


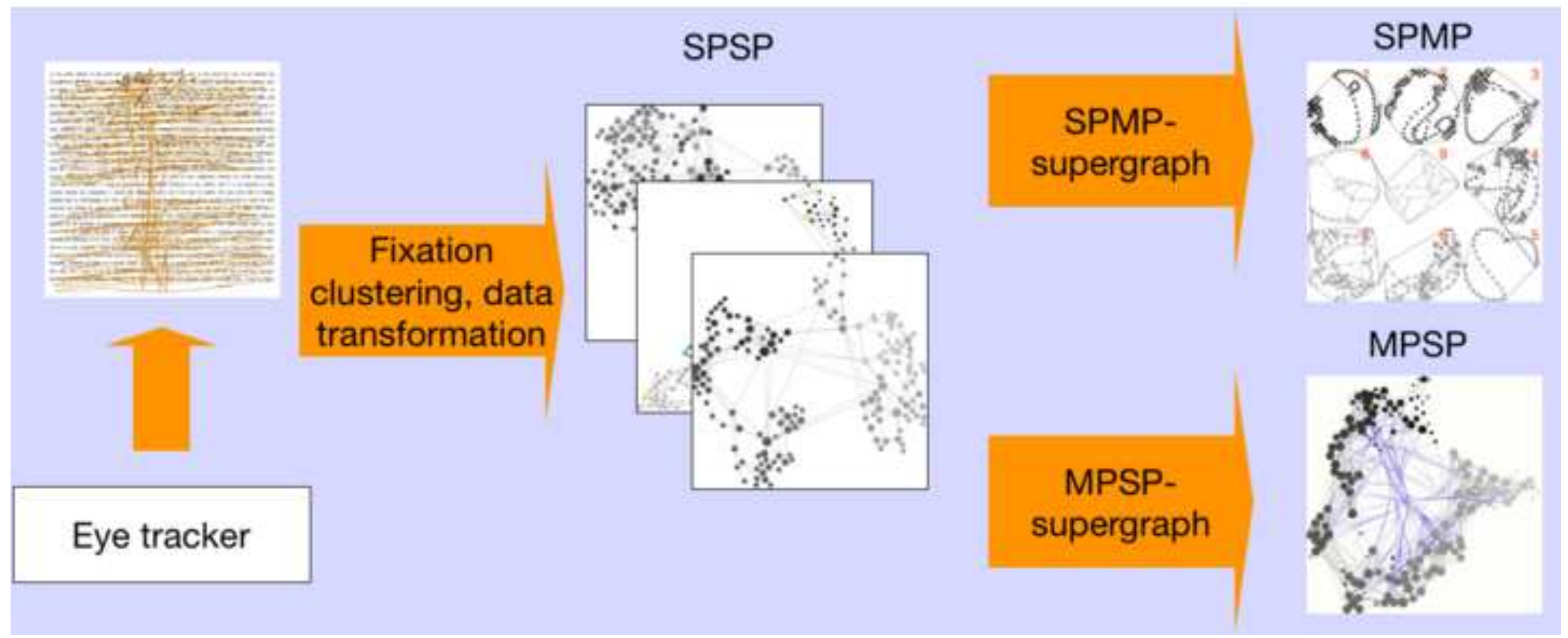

